\title{
Mechanistic insight into the interaction of gastrointestinal mucus with oral diblock copolymers synthesized via ATRP method
}

This article was published in the following Dove Press journal: International Journal of Nanomedicine

\author{
Jiao Liu',* \\ Jie Caol,* \\ Jianhua $\mathrm{Cao}^{2}$ \\ Shangcong Han' \\ Yan Liang' \\ Mingfeng $\mathrm{Bai}^{3}$ \\ Yong Sun'
}

'Department of Pharmaceutics, School of Pharmacy, Qingdao University, Qingdao, China; ${ }^{2}$ Department of Pharmacy, Qingdao No 3 Hospital, Qingdao, China; ${ }^{3}$ Department of Radiology and Radiological Sciences, Institute of Imaging Sciences,

Vanderbilt University Medical Center, Nashville, TN, USA

*These authors contributed equally to this work
Correspondence: Yong Sun

Department of Pharmaceutics, School of Pharmacy, Qingdao University, No 38 Dengzhou Road, Qingdao 26602I, China Tel +86 5328299 I 203 Email sunyong@qdu.edu.cn
Introduction: Nanoparticles are increasingly used as drug carriers for oral administration. The delivery of drug molecules is largely dependent on the interaction of nanocarriers and gastrointestinal (GI) mucus, a critical barrier that regulates drug absorption. It is therefore important to understand the effects of physical and chemical properties of nanocarriers on the interaction with GI mucus. Unfortunately, most of the nanoparticles are unable to be prepared with satisfactory structural monodispersity to comprehensively investigate the interaction. With controlled size, shape, and surface chemistry, copolymers are ideal candidates for such purpose.

Materials and methods: We synthesized a series of diblock copolymers via the atom transfer radical polymerization method and investigated the GI mucus permeability in vitro and in vivo. Results: Our results indicated that uncharged and hydrophobic copolymers exhibited enhanced GI absorption.

Conclusion: These results provide insights into developing optimal nanocarriers for oral administration.

Keywords: absorption barriers, oral drug delivery system, ATRP, nanoparticles

\section{Introduction}

Oral administration, because of its convenience, high safety, and good patient compliance, ${ }^{1}$ has played a dominant role in pharmaceutical market for a long time. Oral drug delivery is less invasive compared to injections, which helps to improve patient compliance..$^{2-4}$ However, there is a layer of mucus barrier on the surface of the gastrointestinal (GI) tract. ${ }^{5-7}$ On the one hand, it serves as a very efficient barrier that provides lubrication and regulates moisture content in the underlying tissues, as well as prevents harmful particles, viruses, and bacteria from entering the human body. ${ }^{8,9}$ On the other hand, the protective function of the mucus also hampers the diffusion of drugs, which dramatically reduces their efficiency. ${ }^{10,11}$ Nanoparticles that are unable to contact with the GI epithelial cells during the effective absorption time will be excreted in the stool with updated mucus, resulting in failed drug delivery. ${ }^{12,13}$ Therefore, effective interaction with GI mucosa is crucial for nanocarriers.

Mucus, a complex network of highly branched glycoproteins, lipids, serum macromolecules, electrolytes, cells, and other cellular debris, is the first barrier that orally delivered drugs diffused before entering the circulatory system. ${ }^{14}$ The mucus gel layer, covering the surfaces of mucosal membranes, is a dynamic system, which can be continuously reformed through secretion of mucins by the goblet cells. ${ }^{15}$ Furthermore, the abundance of intermolecular interactions, including hydrogen bonding and electrostatic 
and hydrophobic interactions, coupled with the high degree of physical entanglements between mucus constituents result in the high viscoelasticity and effective porosity of the gel network. ${ }^{16-18}$ The high viscoelasticity and adhesivity of mucus, along with the abundance of possible interactions between particle and environment, can severely retard diffusion of a drug carrier or molecule. ${ }^{19,20}$ Therefore, the physical and chemical properties of nanoparticles, such as charge, hydrophobicity, and particle size, are expected to greatly affect the permeability of nanoparticles through the mucus layer.

The transport of drug carriers across the mucosal barrier has significant implications for the development of oral drug delivery systems. ${ }^{21,22}$ The ability of different materials, such as some polymers, to adhere and diffuse on the surface of mucous coat has been often used in transmucosal delivery system to improve drug bioavailability. ${ }^{16,17}$ Even though efforts have been made in studying the effects of nanoparticle charge, size, and hydrophobicity on diffusion through mucus diffusion, there is a large variation in techniques. ${ }^{23-31}$ Besides, the nanoparticles prepared by using the conventional method often had a range of sizes; therefore, it is difficult to determine the influence of the surface properties on the mucus layer. In addition, the investigations on the effects of particle size, surface charge, and hydrophilicity had little or no consideration on maintaining other effect factors constant. Therefore, in this study, to better understand the interaction of nanocarriers with GI mucus, we synthesized diblock copolymers by atom transfer radical polymerization (ATRP) ${ }^{32-34}$ method. The particle size, surface charge, and hydrophilicity could be precisely controlled by adjusting the reaction parameters. A hydrophobic near-infrared dye cypate, served as model drugs, was encapsulated into the copolymers. Compared with other drug loaded nanoparticles, the permeability of nanoparticles with different properties in GI mucus layer was studied systematically, which provided a reference for the selection of vectors in oral drug delivery system. In addition, most of the previous studies ${ }^{13,18,27}$ on the oral administration system were used for hydrophilic drugs, such as insulin, and our polymer was designed for carrying hydrophobic drugs.

\section{Materials and methods Materials}

Ethyl 2-bromoisobutyrate (EbiB), N,N,N',N",N"-pentamethy ldiethylenetriamine (PMDETA), ethyl methacrylate (EMA), 2-hydroxyethyl methacrylate (HEMA), 2-hydroxyethyl acrylate (HEA), tertiary-butyl Methacrylate (TBMA), dimethylaminoethyl methacrylate (DMAEMA), trifluoroacetic acid (TFA), and CuBr were all purchased from Macklin (Shanghai, China). The chemicals were used without further purification except for $\mathrm{CuBr}$, which was purified by successively washing with glacial acetic acid and ethanol for three times, followed by drying under vacuum at $25^{\circ} \mathrm{C}$ for $12 \mathrm{~h}$. Mucin from porcine stomach (type II) was purchased from Sigma-Aldrich Co. (St Louis, MO, USA). Coumarin 6 was purchased from Aladdin Industrial Corporation (Shanghai, China). N,NDimethylformamide (DMF) and tetrahydrofuran (THF) were all obtained from Sinopharm Chemical Reagent Co (Shanghai, China). Near-infrared dye cypate (Ex: $780 \mathrm{~nm}$, Em: $810 \mathrm{~nm}$ ) was synthesized in our laboratory. Dulbecco's Modified Eagle's Medium, penicillin, streptomycin, trypsin, and fetal bovine serum were purchased from Kangyuan Corporation (Tianjin, China). 3-(4,5-Dimethylthialzol-a-yl)2,5-diphenyltetrazolium bromide (MTT) was purchased from Solarbio Corp (Beijing, China). Kunming normal mice (male) were purchased from Daren Laboratory Animal Co. Ltd. (Qingdao, China).

\section{Ethical statement}

All the animal experiments were performed in the Animal Center of Qingdao University in accordance with National Institutes of Health Animal Care guidelines and the Animal Management Rules of the Ministry of Health of the People's Republic of China. The in vivo studies were approved by the Animal Care Ethical Committee of Qingdao University.

\section{Methods}

\section{Synthesis of the macroinitiator-poly ethyl} methacrylate (PEMA)

In this study, the macroinitiator was synthesized via ATRP. ${ }^{32-34}$ Briefly, EbiB (63 $\mu \mathrm{L}, 0.2576 \mathrm{mmol})$, EMA (1,612 $\mu \mathrm{L}$, $12.88 \mathrm{mmol})$, and PMDETA $(200 \mu \mathrm{L}, 0.9579 \mathrm{mmol})$ were dissolved in $2 \mathrm{~mL}$ DMF. CuBr was quickly added to the reaction tube as the catalyst after the mixture was frozen in liquid nitrogen. Then, the air in the reaction tube was removed by vacuum, and the reaction tube was sealed. The reaction was carried out at $60^{\circ} \mathrm{C}$. After $24 \mathrm{~h}$, the mixture was exposed to air to terminate the reaction. The crude product was precipitated with excess water and then redissolved in DMF. The solution was dialyzed against deionized water for 2 days to remove impurities. The final product was obtained through freeze-drying. Thus, the copolymer PEMA was synthesized.

\section{Synthesis of diblock copolymers}

Synthesis of diblock copolymers with different properties The ATRP of different copolymers were conducted by using purified PEMA as the macroinitiator. And the procedure is similar to that of ATRP of PEMA. First, PEMA 
(0.2 g, $0.058 \mathrm{mmol})$, DMAEMA (492 $\mu \mathrm{L}, 2.92 \mathrm{mmol})$, and PMDETA $(200 \mu \mathrm{L}, 0.9579 \mathrm{mmol})$ were dissolved in $2 \mathrm{~mL}$ of DMF. Through the operating process as above, the positively charged copolymers PEMA-b-PDMAEMA (poly [2-(dimethylamino) ethyl methacrylate]) were obtained by freeze-drying. The diblock copolymers PEMA-b-PHEA (poly [2-hydroxyethyl acrylate]), PEMA-b-PHEMA (poly [2-hydroxyethyl methacrylate]), and PEMA-(PDMAEMAco-PHEA) were prepared by using the same methods. Negatively charged copolymers (PEMA-b-PMAA [poly (methacrylic acid)]) were obtained by treating PEMA-bPTBMA (poly [tertiary-butyl methacrylate]) with TFA for $8 \mathrm{~h}$, followed by dialysis and freeze-drying. The syntheses of all diblock copolymers are depicted in Scheme 1.

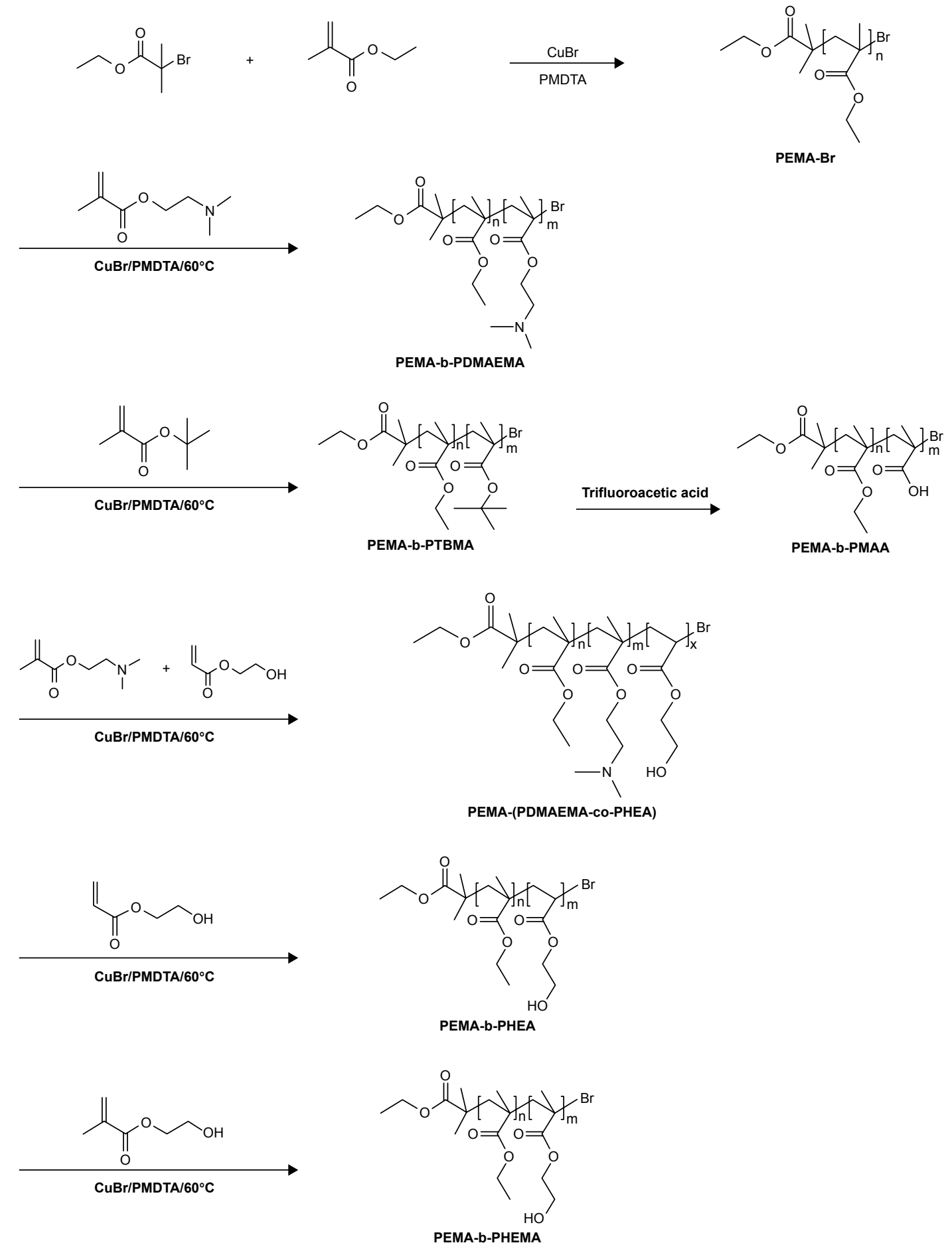

Scheme I Synthetic routes of PEMA-Br polymer, PEMA-b-PDMAEMA, PEMA-b-PMAA, PEMA-(PDMAEMA-co-PHEA), PEMA-b-PHEA and PEMA-b-PHEMA diblock copolymers. 


\section{Synthesis of diblock copolymers with different sizes}

The copolymers with different sizes were synthesized by adjusting the molar ratio of hydrophobic and hydrophilic segments of PEMA-b-PMAA. The hydrophobic segments were synthesized with three molar ratios of 1:30, 1:50, and 1:100, and then the hydrophilic segments were synthesized with the molar ratios of $1: 60,1: 50$, and 1:100, respectively.

\section{Model drug encapsulation}

To investigate the drug loading profiles of the copolymers, fluorescence dye, cypate, served as model drug, was encapsulated into different polymers. Briefly, cypate was first dissolved in $0.5 \mathrm{~mL}$ dimethyl sulfoxide (DMSO) and then added dropwise to $5 \mathrm{~mL}$ phosphate-buffered saline (PBS) (containing $2 \mathrm{mg}$ polymer) under magnetic stirring at room temperature. The solution was then placed into a dialysis tube (MWCO 10000) against $2 \mathrm{~L}$ distilled water for $24 \mathrm{~h}$. After dialysis, centrifugation (10,000 rpm for $10 \mathrm{~min}$ ) was carried out to remove free drugs. Then, particle size and $\zeta$-potential were determined by Malvern Zetasizer NanoZS90. The entrapment efficiency was calculated according to the following equation: entrapment efficiency $=$ (mass of dye loaded in copolymer solution/mass of dye fed initially) $\times$ $100 \%$; loading content $=($ mass of cypate loaded in copolymer solution/mass of cypate-loaded copolymer solution) $\times 100 \%$.

\section{Characterization of diblock copolymers}

To determine the structure of copolymers, the ${ }^{1} \mathrm{H}$ nuclear magnetic resonance ( ${ }^{1} \mathrm{H}$ NMR, JNM-ECP600; JEOL, Tokyo, Japan) spectra and gel permeation chromatography (GPC, PL1110-6504; Agilent Technologies, Santa Clara, CA, USA) of copolymers were measured. The molecular weights and polydispersities of diblock copolymers were measured with PLgel $5 \mu \mathrm{m}$ MIXED-D and a differential refractive index detector. The measurements were performed by using THF as the eluent at a flow rate of $1.0 \mathrm{~mL} / \mathrm{min}$. The calibration was carried out with polystyrene standards. The copolymers were dissolved in THF before being added into water dropwise. The concentration of the polymer solutions was then adjusted to $0.2 \mathrm{mg} / \mathrm{mL}$, and the particle size and $\zeta$-potential were determined by Malvern Zetasizer Nano-ZS90 (Malvern Instruments, Malvern, UK) after THF was evaporated completely. The size and morphologies of polymer solutions were observed by transmission electron microscope (TEM, JEM2010, JEOL). The surface hydrophobicity was evaluated by measuring the contact angle. For the water contact angle (WCA) tests, $5 \mathrm{~mL}$ of the copolymer solution was applied onto a glass microscope slide. The solution formed a thin film on the glass after water was evaporated. And then the WCAs were measured. Furthermore, in order to determine the stability of the samples, the mean particle size and $\zeta$-potential of each sample were measured at different time.

\section{Mucus-copolymer interaction study}

The interaction between copolymers with various properties and artificial mucus was studied by following the procedure below: First, $100 \mu \mathrm{L}$ of artificial mucus $(20 \mathrm{mg} / \mathrm{mL}$ in PBS, $0.1 \mathrm{M}, \mathrm{pH}=6.5$ ) was added into $1 \mathrm{~mL}$ of polymer solutions $(0.2 \mathrm{mg} / \mathrm{mL})$. The mixture was then incubated in a thermomixer at $37^{\circ} \mathrm{C}$ and $120 \mathrm{rpm}$ for $4 \mathrm{~h}$. Next, the mixture was centrifuged at $4^{\circ} \mathrm{C}$ and $12,000 \mathrm{rpm}$ for $30 \mathrm{~min}$. At each point of $1,2,3$, and $4 \mathrm{~h}$, the particle size and $\zeta$-potential were determined after the mixture was dispersed in $1 \mathrm{~mL}$ of distilled water. At the same time, the particle size and $\zeta$-potential of plain mucus were measured as the control $(n=3)$.

\section{Diffusion studies using Transwell ${ }^{\circledR}$ chambers}

To investigate the transportation of copolymers across the mucus barrier, 12-well plates (12 $\mathrm{mm}$; pore size: $3 \mu \mathrm{m}$; Corning Incorporated, Corning, NY, USA) were used as previously reported. ${ }^{35,36}$ First, coumarin-6 was added to copolymer THF solutions to achieve coumarin-6-loaded polymer solutions. In the coumarin-6-loaded polymer solutions, the coumarin-6/copolymer ratio was 1:1,000 (w/w). And then the encapsulation efficiency (EE) of coumarin-6 was measured. $100 \mu \mathrm{L}$ of artificial mucus $(20 \mathrm{mg} / \mathrm{mL}$ in PBS, $0.1 \mathrm{M}, \mathrm{pH}=6.5$ ) was put in each Transwell and $500 \mu \mathrm{L}$ of the coumarin-6-loaded polymer solutions $(0.2 \mathrm{mg} / \mathrm{mL})$ were placed on the top of the mucus layer. The Transwell was put in 12-well plate, and the acceptor chamber contained $1.5 \mathrm{~mL}$ of PBS ( $\mathrm{pH}=6.5$ ). The plate was put on the incubated shakers (WIGGENS, WS-600) at $37^{\circ} \mathrm{C}$ and $120 \mathrm{rpm}$. At predetermined time points of $1,2,3,4,5$, and $6 \mathrm{~h}, 100 \mu \mathrm{L}$ samples were removed from the acceptor chamber and replaced with the same volume of PBS. The amount of permeated particles was calculated by standard curve of coumarin- 6 concentration and fluorescence intensity $(n=3)$.

\section{In vitro cytotoxicity assays}

MTT assay was used to test the cytotoxicity of different copolymers on Caco- 2 cells. Cells were seeded in 96-well plates at a density of $2 \times 10^{3}$ per well and incubated for $24 \mathrm{~h}$ at $37^{\circ} \mathrm{C}$ before starting the treatment. Prior to the test, the medium was withdrawn and the cells were washed with PBS. Subsequently, cells were treated with a series of 
different concentrations (from 5 to $400 \mu \mathrm{g} / \mathrm{mL}$ ) of different copolymer solutions. After incubation for $24 \mathrm{~h}, 20 \mu \mathrm{L}$ of MTT $(5 \mathrm{mg} / \mathrm{mL})$ was added to each cell and incubated for another $4 \mathrm{~h}$. Then the medium was removed, the cells were washed by PBS once and replaced by $150 \mu \mathrm{L}$ of DMSO. Finally, the absorbance was measured by a microplate reader (Thermo Fisher Scientific, Waltham, MA, USA) at $490 \mathrm{~nm}$. The Caco-2 cells treated with the medium were evaluated as control. Cell viability was calculated by the following formula:

$$
\text { Cell viability }(\%)=\frac{A_{\text {treated }}}{A_{\text {control }}} \times 100
$$

where $\mathrm{A}_{\text {treated }}$ and $\mathrm{A}_{\text {control }}$ represented the absorbance of treated cells and control cells, respectively.

\section{In vitro distribution in the small intestine}

Copolymers labeled with coumarin-6 were prepared for distribution studies. To determine the distribution of coumarin-6labeled copolymers in the small intestine, the intestinal loop experiments were carried out as previously described. ${ }^{37}$ First, Kunming mice were sacrificed, and a $2 \mathrm{~cm}$ jejunum region was taken out from the abdomen. Then the jejunum was tied off, and $200 \mu \mathrm{L}$ of coumarin-6-labeled polymer solutions was injected into the loop using a syringe. At the completion of the experiment (about $30 \mathrm{~min}$ ), the tissues were sliced and $1 \mathrm{~cm}$ segment of tissues were placed in chambers. Sections were imaged by using a confocal microscopy (Nikon A1R MP; Nikon Corporation, Tokyo, Japan).

\section{In vivo distribution studies}

In order to investigate the absorption and excretion of copolymers with various properties in the GI tract, 6-8 week male Kunming mice were used. First, cypate-uploaded polymer solutions $(0.2 \mathrm{mg} / \mathrm{mL})$ were prepared and the cypate/ copolymer ratio was 1:10. The mice were weighed, and then the abdominal hair was shaved before Vaseline was applied. The mice were divided into four groups, which were administered with different kinds of copolymers $(150 \mu \mathrm{L}$ per $10 \mathrm{~g}$ weight). The mice were fasted for $12 \mathrm{~h}$ before gavage administration. At predetermined time points of $0.5,1,2,4,8$, and $12 \mathrm{~h}$, fluorescence intensity was observed by using in vivo imaging system (IVIS Spectrum, Ex: 780 nm, Em: 810 nm; PerkinElmer Inc., Waltham, MA, USA). Then, the mice were sacrificed before dissecting the stomach and intestine, and ex vivo fluorescence imaging was carried out.
In addition, the release of copolymer-cypate in vitro was calculated to ensure that changes of fluorescence intensity in vivo was caused by copolymer-cypate rather than the free cypate. Briefly, the sample was kept inside a dialysis bag and dispersed in PBS ( $\mathrm{pH}=7.4$ ) before shaking at $100 \mathrm{rpm}$. Then, $200 \mu \mathrm{L}$ of the suspension was withdrawn at predetermined time intervals and replaced with equal volume of solution. Finally, the absorbance of the sample was determined by UV spectrophotometer.

\section{Ex vivo distribution in small intestine}

To further investigate the diffusion of copolymers in the intestine, ex vivo animal experiment was conducted. First, mice were anesthetized by $1 \%$ pentobarbital sodium $(70 \mathrm{mg} / \mathrm{kg})$, and a $2 \mathrm{~cm}$ jejunum region was tied off from an incision in the abdomen. Then $200 \mu \mathrm{L}$ of coumarin-6-loaded polymer solutions was injected into the loop by using a syringe. At different time points (from 15 to $60 \mathrm{~min}$ ), the mice were sacrificed and the tissues were frozen in Optimal Cutting Temperature compound. For all tissues, $20 \mu \mathrm{m}$ thick sections were cut by using a Leica CM-3050-S cryostat (Leica, Wetzlar, Germany). The tissue sections were stained with Mounting Medium, antifading with 4',6-diamidino-2-phenylindole and were imaged by using a confocal microscopy (Nikon A1R MP; Nikon).

\section{Results and discussion Synthesis and characterization of diblock copolymers}

The synthetic routes of diblock copolymers are shown in Scheme 1. According to the charge character, hydrophobicity, and pore size distribution of the mucus, ${ }^{23,25}$ we designed different diblock copolymers to investigate their behavior in the mucus layer. PEMA-Br was first prepared by ATRP. Then, ATRP of DMAEMA/TBMA/HEA/HEMA was carried out in DMF by using PEMA-Br as a macroinitiator and $\mathrm{CuBr} / \mathrm{PMDETA}$ as the catalyst system. By the method of polymerizing different monomers on the basis of macroinitiator, we synthesized copolymers with different charges and hydrophobicity properties. At the same time, we obtained copolymers with different sizes by adjusting the proportion of the hydrophobic segments and hydrophilic segments of the diblock polymer. Compared to previous investigations, ${ }^{24,32-34}$ polymers could be prepared by controllable polymerization with predictable molecular weights and structures by ATRP method. The polymers with controllable molecular weight, narrow molecular weight distribution, abundant raw materials, and low price were synthesized. 
A<smiles>C=C(C)C(=O)OCC</smiles>

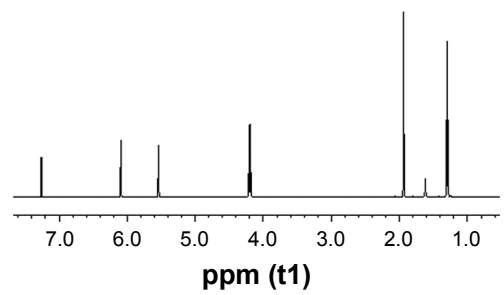

D
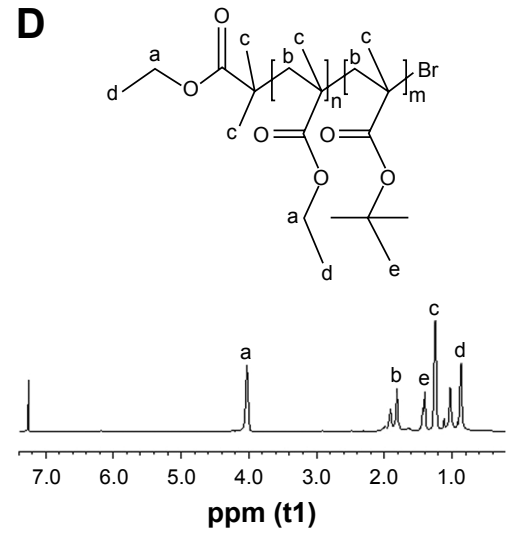

B

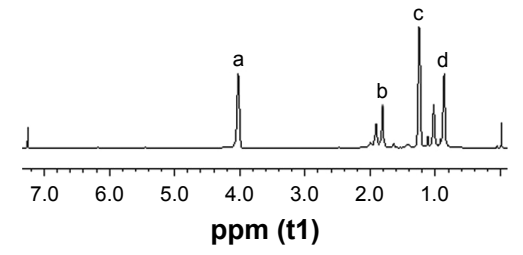

E

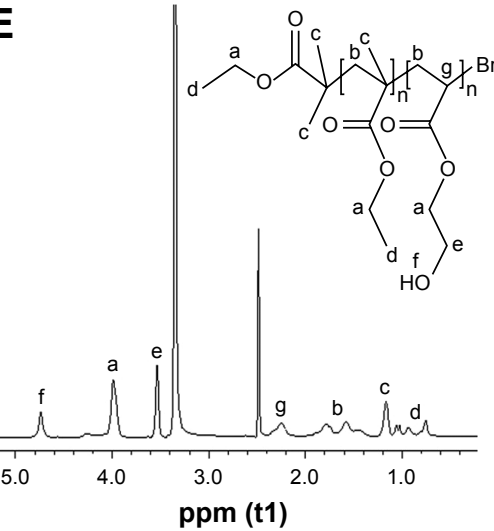

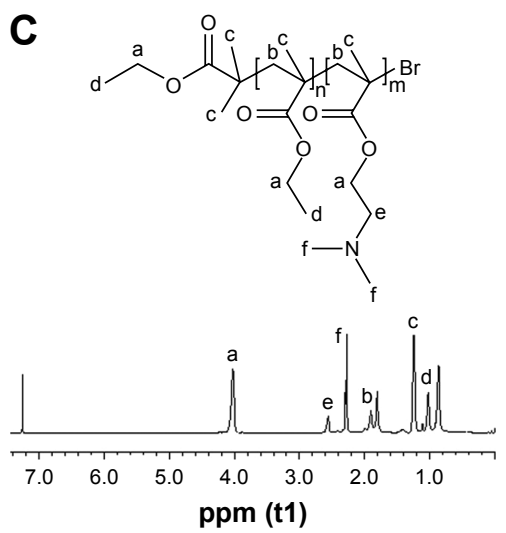

$\mathbf{F}$

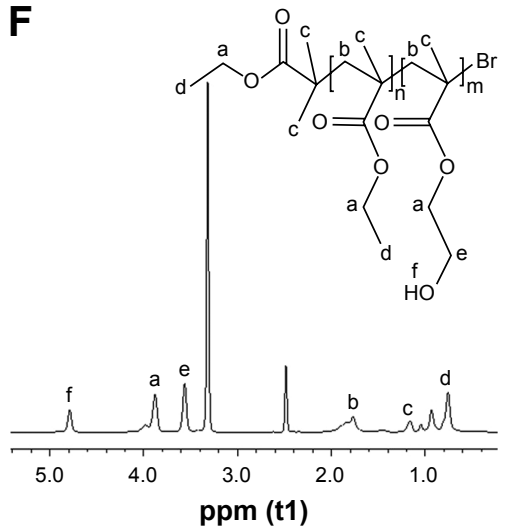

Figure I 'H NMR spectra of (A) ethyl methacrylate, (B) PEMA-Br polymer, (C) PEMA-b-PDMAEMA diblock copolymer, (D) PEMA-b-PTBMA diblock copolymer, (E) PEMA-bPHEA diblock copolymer, and (F) PEMA-b-PHEMA diblock copolymer. $\mathrm{CDCl}_{3}$ was used as the solvent in all images except (E) and (F) which were dissolved in DMSO- $\mathrm{d}_{6}$. Abbreviations: 'H NMR, 'H nuclear magnetic resonance; PEMA, poly ethyl methacrylate; PDMAEMA, poly (2-[dimethylamino] ethyl methacrylate); PTBMA, poly (tertiarybutyl methacrylate); PHEA, poly (2-hydroxyethyl acrylate); PHEMA, poly (2-hydroxyethyl methacrylate); DMSO, dimethyl sulfoxide.

${ }^{1} \mathrm{H}$ NMR and GPC were used to confirm the chemical structures and molecular weights of the copolymers. Figure 1B shows the ${ }^{1} \mathrm{H}$ NMR spectrum of PEMA-Br sample: resonances at $\delta 0.86-1.02 \mathrm{ppm}$ (peak d) indicates the presence of pendent methyl $\left(-\mathrm{COOCH}_{2} \mathrm{CH}_{3}\right)$ protons, whereas the peak at $\delta 4.02$ (peak a) corresponds to methylene $\left(-\mathrm{COOCH}_{2} \mathrm{CH}_{3}\right)$ protons. In the region of $\delta 1.81-1.25$, the peaks at $\delta 1.81-1.99 \mathrm{ppm}$ (peak b) are assigned to the methylene protons $\left[-\mathrm{CH}_{2} \mathrm{C}\left(\mathrm{CH}_{3}\right)-\right]$ and the peaks at $\delta$ $1.25 \mathrm{ppm}$ (peak c) are attributed to methyl $\left[-\mathrm{CH}_{2} \mathrm{C}\left(\mathrm{CH}_{3}\right)-\right]$ from PEMA backbone. Comparing the ${ }^{1} \mathrm{H}$ NMR spectra of ethyl methacrylate (Figure 1A) with that of PEMA-Br (Figure 1B) in $\mathrm{CDCl}_{3}$, we found that the peaks corresponding to the double bond protons ( $\delta 5.54$ and $\delta 6.09)$ disappeared and new peaks at $\delta 1.81-1.99 \mathrm{ppm}$ (peak b) appeared in Figure 1B, indicating that all the double bonds were reacted. Figure $1 \mathrm{C}$ shows the ${ }^{1} \mathrm{H}$ NMR spectrum of PEMA-b-PDMAEMA. The chemical shifts, $\delta 2.56 \mathrm{ppm}$ (peake) and $\delta 2.27 \mathrm{ppm}$ (peak f), are mainly attributed to the pendent methylene $\left[-\mathrm{COOCH}_{2} \mathrm{CH}_{2}-\mathrm{N}\left(\mathrm{CH}_{3}\right)_{2}\right]$ and methyl
$\left[-\mathrm{COOCH}_{2} \mathrm{CH}_{2}-\mathrm{N}\left(\mathrm{CH}_{3}\right)_{2}\right]$ protons of the DMAEMA arms, respectively, indicating the presence of DMAEMA chains. The signal at $1.40 \mathrm{ppm}$ (peak e) of Figure 1D was assigned to the pendent methyl $\left[-\mathrm{COOC}\left(\mathrm{CH}_{3}\right)_{3}\right]$ protons, which confirmed that PEMA-b-PTBMA was formed. The new singlets of $\delta 3.56$ (peak e) and $\delta 4.78$ (peak f) in Figure $1 \mathrm{~F}$ were assigned to pendent methylene $\left(-\mathrm{COOCH}_{2} \mathrm{CH}_{2} \mathrm{OH}\right)$ protons and pendent hydroxy $\left(-\mathrm{COOCH}_{2} \mathrm{CH}_{2} \mathrm{OH}\right)$ protons, indicating the new group in copolymers. In comparison with the spectrum shown in Figure 1F, the new singlet of $\delta 2.25$ (peak g) shown in Figure 1E (PEMA-b-PHEA) corresponds to methine $\left[-\mathrm{CH}_{2} \mathrm{CH}-\right]$ protons, indicating the presence of HEA chains. These results confirmed the presence of hydrophilic and hydrophobic groups in the copolymers.

Figure 2 and Table 1 showed the GPC data of five different copolymers. The different diblock copolymers all exhibited a higher molecular weight compared with PEMA-Br homopolymer, indicating that the ATRP reaction was successfully achieved. From Table 1, it can be seen that PEMA-Br is polymerized from small molecules into large molecules. 


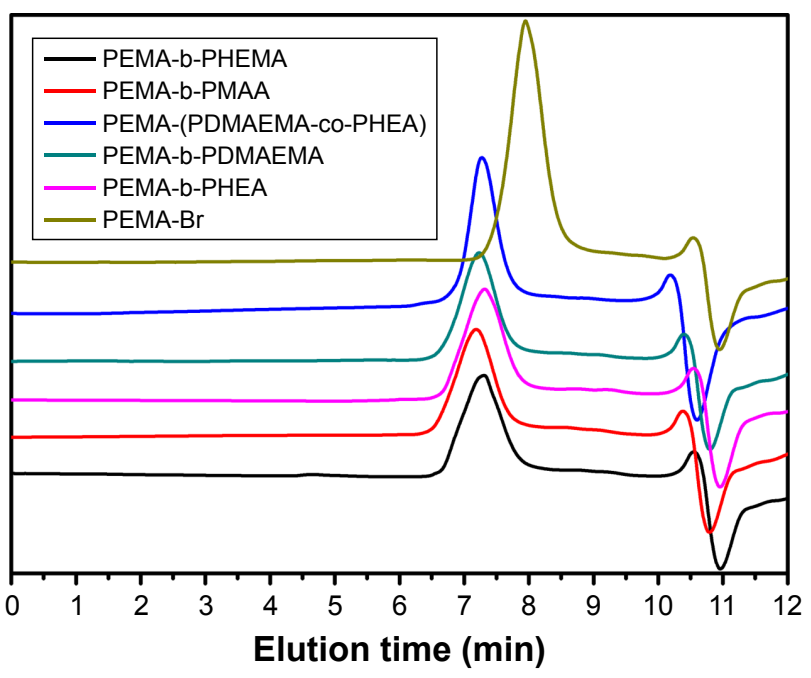

Figure 2 Molecular weight distribution of each polymer.

Note: Signals at 10.3 and 10.8 min of elution time were caused by solvent.

Abbreviations: PEMA, poly ethyl methacrylate; PDMAEMA, poly (2-[dimethylamino] ethyl methacrylate); PHEA, poly (2-hydroxyethyl acrylate); PHEMA, poly (2-hydroxyethyl methacrylate); PMAA, poly (methacrylic acid).

As compared to PEMA-Br, the molecular weight of other copolymers became much larger, suggesting the occurrence of polymerization.

\section{Particle size, $\zeta$-potential, and contact angle} As expected, the five copolymers (PEMA-b-PDMAEMA, PEMA-b-PMAA, PEMA-[PDMAEMA-co-PHEA], PEMAb-PHEA, and PEMA-b-PHEMA) showed roughly the same particle size of around $200 \mathrm{~nm}$ (Table 2), and TEM images indicated small and uniform size (Figure 3A-E). For the $\zeta$-potential, it can be altered by changing the ligand of the copolymers. It is obvious that when the hydrophilic segment was DMAEMA, the copolymers carried a positive charge on particle surface $(+47.7 \mathrm{mV})$, while PEMA-b-PMAA showed a negative potential $(-49.9 \mathrm{mV})$ and PEMA-(PDMAEMA-co-PHEA) was electrically neutral

Table I Characteristic data of GPC

\begin{tabular}{llll}
\hline Sample & $\mathbf{M}_{\mathbf{n}}$ & $\mathbf{M}_{\mathbf{w}}$ & PD \\
\hline PEMA-Br & 3,999 & $4,81 \mathrm{I}$ & 1.203 \\
PEMA-b-PDMAEMA & 10,399 & 12,884 & 1.229 \\
PEMA-b-PMAA & 10,843 & 13,391 & 1.235 \\
PEMA-(PDMAEMA-co-PHEA) & 9,840 & 11,985 & 1.218 \\
PEMA-b-PHEA & 9,340 & 11,610 & 1.243 \\
PEMA-b-PHEMA & 9,670 & 12,039 & 1.245 \\
\hline
\end{tabular}

Note: Number-average molecular weight $\left(M_{n}\right)$, weight-average molecular weight $\left(M_{w}\right)$, polydispersion index, $P D=M_{w} / M_{n}$.

Abbreviations: GPC, gel permeation chromatography; PEMA, poly ethyl methacrylate; PDMAEMA, poly (2-[dimethylamino] ethyl methacrylate); PHEA, poly (2-hydroxyethyl acrylate); PHEMA, poly (2-hydroxyethyl methacrylate); PMAA, poly (methacrylic acid).
(Table 2). Our approach easily offered three materials with different electric charges.

Different copolymer sizes were obtained by adjusting the ratio of hydrophobic segment and hydrophilic segment, and three polymers of different particle sizes were synthesized. The materials were characterized by particle size and TEM. Figure $3 \mathrm{~F}-\mathrm{H}$ shows the particle sizes ranging from 150 to $500 \mathrm{~nm}$, and the three materials were named A, B, and $\mathrm{C}$, respectively. In addition, to evaluate the stability of the polymers, changes in the mean particle size and $\zeta$-potential of the nanoparticles at different times were measured. No significant changes in the mean particle size or $\zeta$-potential of the polymers were found after nearly 1 month, suggesting that most of the particles remained intact (Figure S1).

Wetting behavior of the copolymers was assessed by measuring the contact angle (Figure 3I and J). The contact angle of the drop was found to be $86.7^{\circ} \pm 3.32^{\circ}$ and $62.6^{\circ} \pm 3.81^{\circ}$ for PEMA-b-PHEMA and PEMA-b-PHEA, respectively. From the data, we can conclude that the two materials are both hydrophilic. HEA significantly enhanced the surface hydrophilicity of the copolymers than HEMA, indicating that HEMA is more hydrophobic than HEA. Therefore, compared to PEMA-b-PHEA, PEMA-b-PHEMA is less hydrophilic. In order to distinguish these two materials, PEMA-b-PHEA was named hydrophilic material, while PEMA-b-PHEMA was named hydrophobic material. The results indicated that by using ATRP method, we can easily obtain polymers with different potentials, hydrophilicity, and particle sizes, which provided better materials for the in vivo and in vitro experiments.

\section{Model drug encapsulation}

After encapsulating with hydrophobic dye cypate, the average diameter by DLS measurement all decreased (Table 2). The hydrophobic interaction between cypate and polymer would result in the hydrophobic core becoming much more intense, representing the decreased size compared to initial polymer solution. Since the cypate was uploaded into the inner core of the polymers, $\zeta$-potential did not show obvious changes, and the $\mathrm{EE}$ is $74.6 \%$.

\section{Mucus-copolymer interaction study}

To determine whether the properties of copolymers remained the same during the interaction with mucus, we monitored the $\zeta$-potential and size of copolymers (Figures 4 and S2). As shown in Figure 4A, mucus can obviously change the 
Table 2 Characteristic data of copolymers and cypate-loaded copolymers

\begin{tabular}{|c|c|c|c|}
\hline Sample & Size $(n m)$ & PDI & $\begin{array}{l}\zeta \text {-Potentia } \\
(\mathrm{mV})\end{array}$ \\
\hline (+) PEMA-b-PDMAEMA & $201.8 \pm 0.78$ & $0.105 \pm 0.036$ & $47.7 \pm 0.624$ \\
\hline (+) PEMA-b-PDMAEMA@cypate & $174.1 \pm 1.905$ & $0.129 \pm 0.021$ & $45.9 \pm 0.583$ \\
\hline PEMA-(PDMAEMA-co-PHEA) & $204.1 \pm 3.024$ & $0.067 \pm 0.04$ & $-2.4 I \pm 0.3 I$ \\
\hline PEMA-(PDMAEMA-co-PHEA)@cypate & $178.4 \pm 1.015$ & $0.125 \pm 0.026$ & $-2.11 \pm 0.255$ \\
\hline (-) PEMA-b-PMAA & $|96.9 \pm 2.6|$ & $0.134 \pm 0.012$ & $-49.9 \pm 0.473$ \\
\hline (-) PEMA-b-PMAA@cypate & $|55.| \pm \mid .75$ & $0.115 \pm 0.013$ & $-50.3 \pm 0.387$ \\
\hline (Hydrophilic) PEMA-b-PHEA & $202.6 \pm 4.53$ & $0.124 \pm 0.039$ & $-19.9 \pm 0.208$ \\
\hline (Hydrophilic) PEMA-b-PHEA@cypate & $182.9 \pm 1.893$ & $0.158 \pm 0.005$ & $-19.1 \pm 0.334$ \\
\hline (Hydrophobic) PEMA-b-PHEMA & $199.5 \pm 2.29$ & $0.133 \pm 0.039$ & $-19.9 \pm 0.529$ \\
\hline (Hydrophobic) PEMA-b-PHEMA@cypate & $169.3 \pm 0.462$ & $0.159 \pm 0.021$ & $-20.8 \pm 0.438$ \\
\hline PEMA $_{30}-$ b-PMAA 60 & $|4| .6 \pm 3.3$ & $0.128 \pm 0.019$ & $-46.5 \pm 0.681$ \\
\hline PEMA $_{30}$-b-PMAA $60 @$ cypate & $124.1 \pm 1.305$ & $0.095 \pm 0.008$ & $-48.2 \pm 0.683$ \\
\hline PEMA $_{50}$-b-PMAA ${ }_{50}$ & $3 \mid 7.1 \pm 4.3$ & $0.143 \pm 0.028$ & $-5|.4 \pm| .7 \mid$ \\
\hline PEMA $_{50}$-b-PMAA 50 @cypate & $282.7 \pm 4.539$ & $0.058 \pm 0.031$ & $-49.8 \pm 0.987$ \\
\hline PEMA $_{100}-\mathrm{b}-\mathrm{PMAA}_{100}$ & $487.3 \pm 26.3$ & $0.251 \pm 0.025$ & $-49.9 \pm 1.47$ \\
\hline PEMA $_{100}$-b-PMAA $_{100} @$ cypate & $468.2 \pm 17.5$ & $0.203 \pm 0.018$ & $-48.8 \pm 1.103$ \\
\hline
\end{tabular}

Abbreviations: PEMA, poly ethyl methacrylate; PDMAEMA, poly (2-[dimethylamino] ethyl methacrylate); PHEA, poly (2-hydroxyethyl acrylate); PHEMA, poly (2-hydroxyethyl methacrylate); PMAA, poly (methacrylic acid); PDI, polydispersion index.
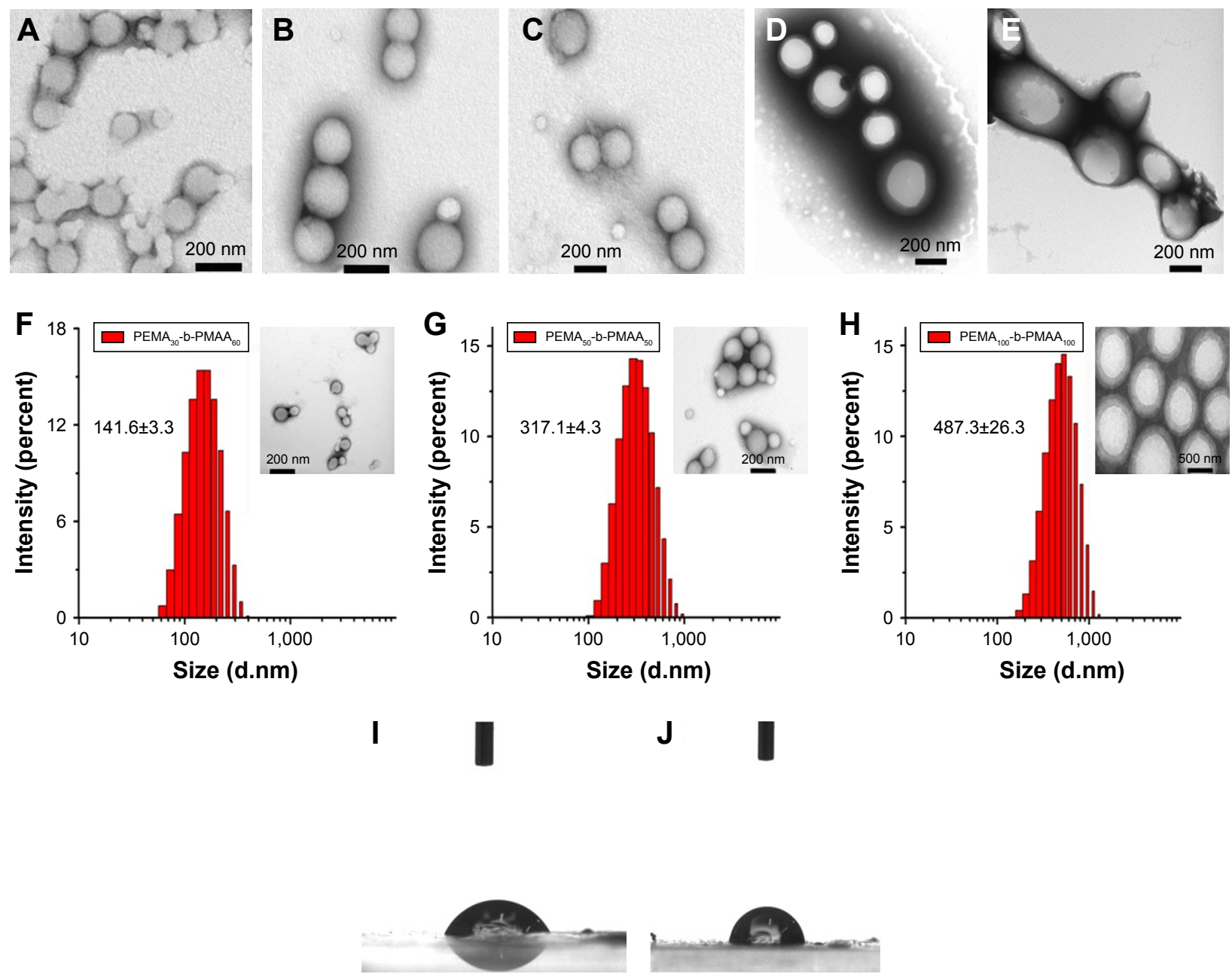

Figure 3 Transmission electron microscope images of (A) PEMA-b-PDMAEMA, (B) PEMA-b-PMAA, (C) PEMA-(PDMAEMA-co-PHEA), (D) PEMA-b-PHEA, and (E) PEMAb-PHEMA polymer solutions and size distribution of $(\mathbf{F})$ PEMA $_{30}$-b-PMAA ${ }_{60},(\mathbf{G})$ PEMA $_{50}$-b-PMAA, ${ }_{50}$, and $(\mathbf{H})$ PEMA $_{100}$-b-PMAA 100 polymer solutions; inset: transmission electron microscope images of three different size polymer solutions; images I and $\mathbf{J}$ show the water contact angles of PEMA-b-PHEA and PEMA-b-PHEMA, respectively. Images were taken at $30 \mathrm{~s}$ after water drops contacting on the surface.

Abbreviations: PEMA, poly ethyl methacrylate; PDMAEMA, poly (2-[dimethylamino] ethyl methacrylate); PHEA, poly (2-hydroxyethyl acrylate); PHEMA, poly (2-hydroxyethyl methacrylate); PMAA, poly (methacrylic acid). 

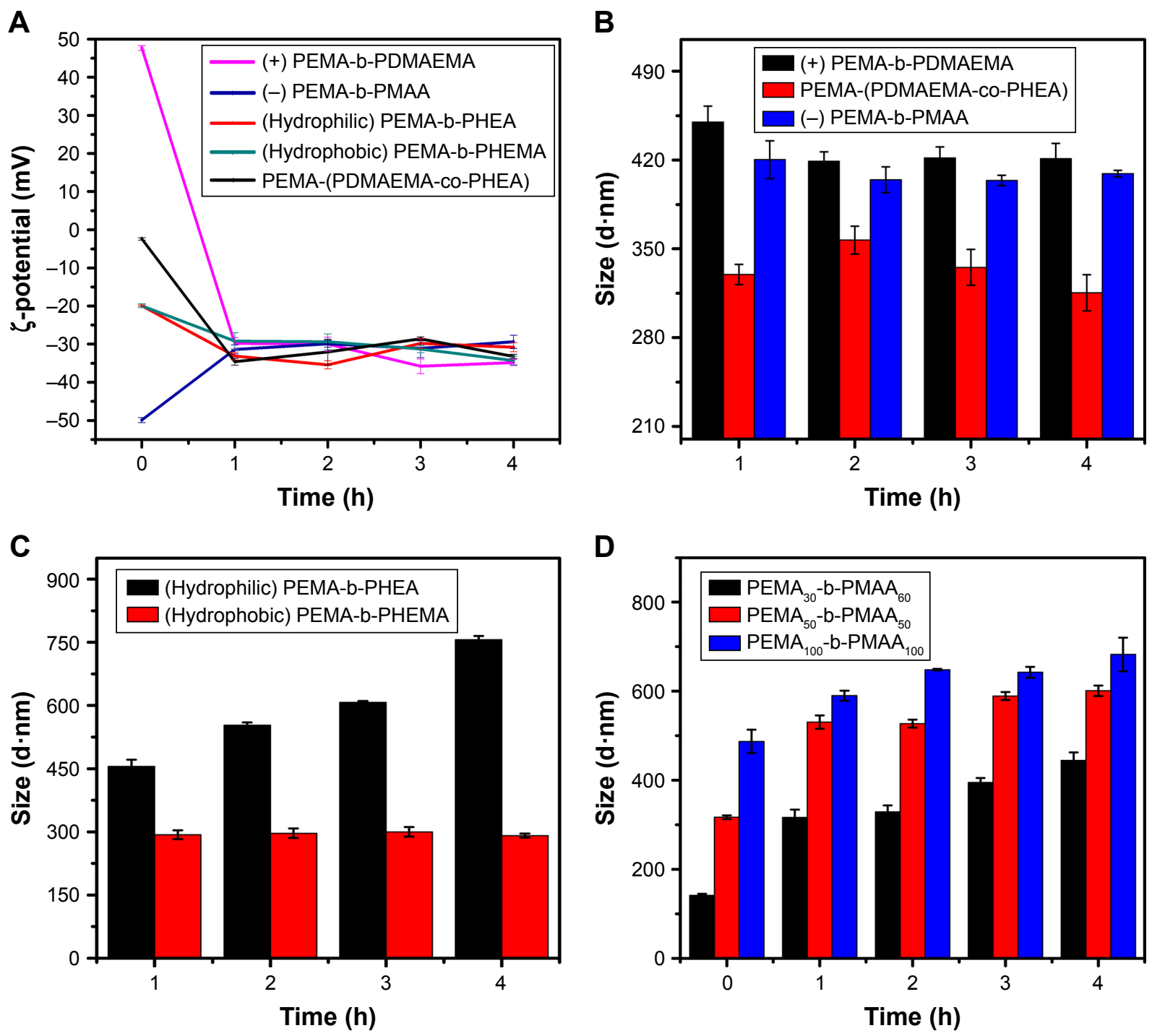

Figure 4 (A) Variation of $\zeta$-potential of five different properties, particle size of (B) PEMA-b-PDMAEMA, PEMA-(PDMAEMA-co-PHEA), and PEMA-b-PMAA and (C) PEMAb-PHEA and PEMA-b-PHEMA, and (D) three different size polymer solutions incubated with mucus at $37^{\circ} \mathrm{C} \mathrm{pH} 6.5$. Values are mean \pm standard deviation ( $\mathrm{n}=3$ ). Abbreviations: PEMA, poly ethyl methacrylate; PDMAEMA, poly (2-[dimethylamino] ethyl methacrylate); PHEA, poly (2-hydroxyethyl acrylate); PHEMA, poly (2-hydroxyethyl methacrylate); PMAA, poly (methacrylic acid)

$\zeta$-potential of PEMA-b-PDMAEMA from positive to negative in $1 \mathrm{~h}$ and the $\zeta$-potential of all the materials changed to roughly $-30 \mathrm{mV}$ after incubation for $4 \mathrm{~h}$. It is obvious that the surface properties of the positively charged materials have changed by the effect of mucus. Figure 4B showed that, compared to the particle size of PEMA-(PDMAEMAco-PHEA), the particle size of positively charged materials increased from 200 to $450 \mathrm{~nm}$ and the size of negatively charged materials increased to $410 \mathrm{~nm}$. For hydrophilic and hydrophobic nanoparticles, the size of PEMA-b-PHEMA was maintained at about $250 \mathrm{~nm}$, whereas the particle size of PEMA-b-PHEA increased to $700 \mathrm{~nm}$ (Figure 4C). Hence, the particle size of PEMA-b-PHEA showed greater change than that of PEMA-b-PHEMA. From the data of particle size and $\zeta$-potential, it can be concluded that the change of hydrophilic and positively charged copolymers is more significant than those of others. In contrast, neutral and hydrophobic copolymers showed little size change, probably due to less aggregation in mucus. It can be seen in Figure 4D that the particle size of copolymers increased significantly after interaction with mucus. Specifically, the particle size of copolymer A increased from 140 to $400 \mathrm{~nm}$, the particle size of copolymer B increased from 317 to $600 \mathrm{~nm}$, and that of copolymer C increased from 500 to $670 \mathrm{~nm}$. Although the size of copolymer A increased at a greater extent, the eventual particle size remained the smallest among these three copolymers. The particle size and $\zeta$-potential of mucus have been measured to be about $1,800 \mathrm{~nm}$ and $-20 \mathrm{mV}$, respectively, suggesting considerable change of $\zeta$-potential and particle size were possibly due to the aggregation of polymers in 
the mucus layer. The adhesion mechanisms of copolymers to mucus are predominantly based on electrostatic/ ionic interactions, as well as hydrophobic interactions or hydrogen bonding. Here, the mucin-copolymer interaction method suggested that positively charged and hydrophilic copolymers have a higher tendency to electrostatically bind mucin as compared to others. The strength of interactions was confirmed by the $\zeta$-potential of the mucin-copolymer dispersion, indicating the penetration of polymers in mucus layer, which we will investigate in another experiment.

\section{Diffusion studies using Transwell ${ }^{\circledR}$ chambers}

Figure $5 \mathrm{~A}$ and $\mathrm{B}$ shows the permeability of polymer with different properties in the mucus layer. The infiltration capacity was calculated by the standard curve of coumarin- 6 , and the
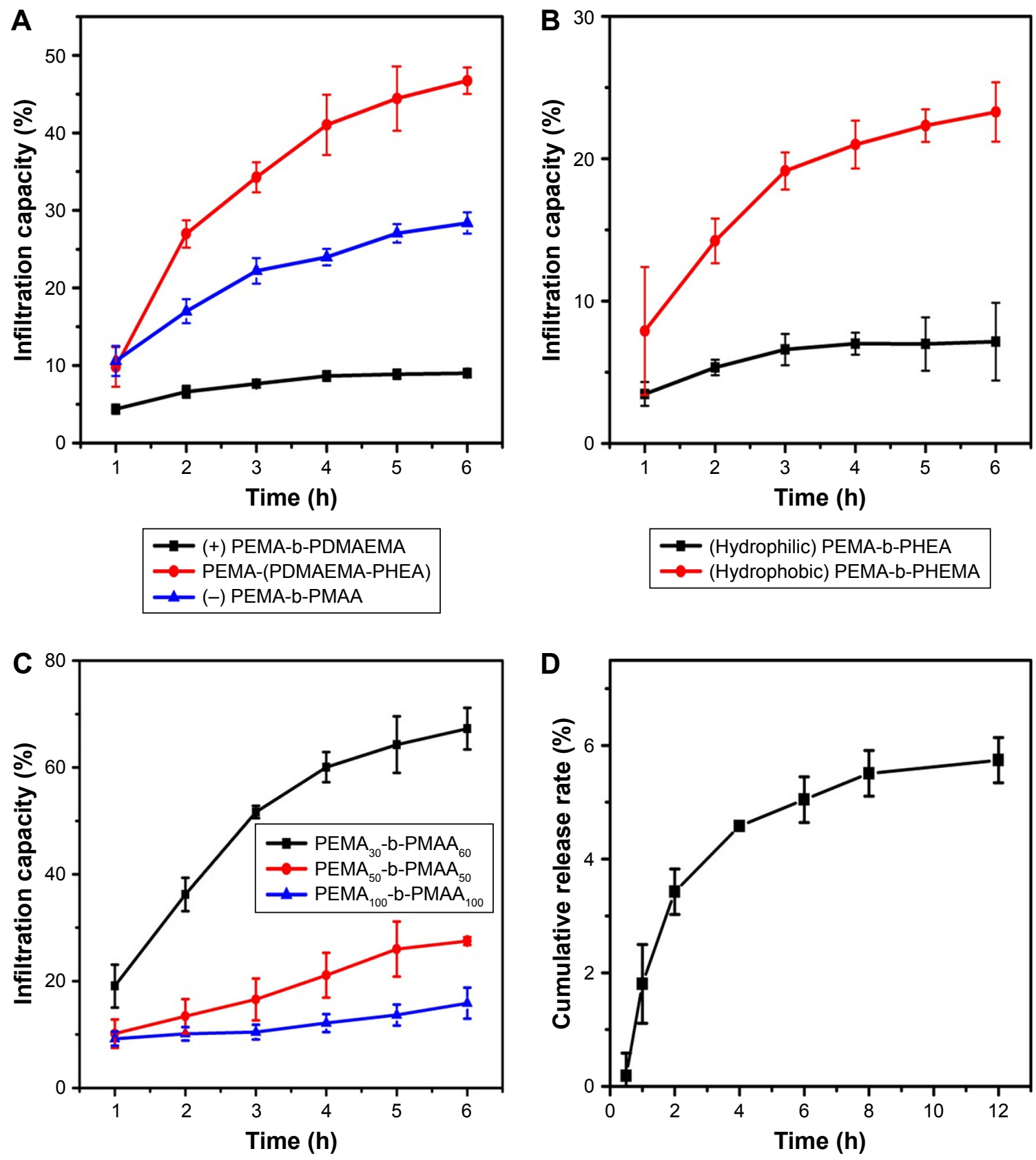

Figure 5 Release of (A) PEMA-b-PDMAEMA, PEMA-(PDMAEMA-co-PHEA), and PEMA-b-PMAA and (B) PEMA-b-PHEA and PEMA-b-PHEMA, and (C) three different size polymer solutions incubated with mucus at $37^{\circ} \mathrm{C} \mathrm{pH} 6.5$ in Transwell ${ }^{\circledR}$ chamber. (D) Comparison of cumulative release rate of copolymer-cypate in vitro. Values are mean \pm standard deviation $(n=3)$.

Abbreviations: PEMA, poly ethyl methacrylate; PDMAEMA, poly (2-[dimethylamino] ethyl methacrylate); PHEA, poly (2-hydroxyethyl acrylate); PHEMA, poly (2-hydroxyethyl methacrylate); PMAA, poly (methacrylic acid). 
EE is about $56 \%$. As shown in Figure $5 \mathrm{~A}$, permeability of negatively charged and uncharged copolymers increased rapidly during the first $2 \mathrm{~h}$, whereas infiltration of positively charged copolymers increased only slightly after $4 \mathrm{~h}$. Neutral copolymers showed the highest permeation, and the infiltration capacity reached as high as $46 \mu \mathrm{g}$. In Figure 5B, the permeability of the hydrophilic copolymers showed no obvious change, with the infiltration capacity of about $7 \mu \mathrm{g}$. The penetration of PEMA-b-PHEMA remained stable after $1 \mathrm{~h}$, with the infiltration capacity of up to $23 \mu \mathrm{g}$ at $6 \mathrm{~h}$. From these data, it can be concluded that compared to charged copolymers, neutral copolymers can more easily penetrate the mucus layer. In addition, it is not easy for the hydrophilic copolymers to penetrate the mucus layer. This result is consistent with the result of "Mucus-copolymers interaction study" section mentioned above. Mucoadhesion studies performed by the mucus-copolymer method confirmed the intrinsic capability of PEMA-b-PDMAEMA and PEMA-b-PHEA to interact with negatively charged mucin, and this finding prompted toward the investigation of permeating ability. Furthermore, the negatively charged and neutral copolymers showed small changes in the particle size and $\zeta$-potential, indicating a large permeation in the mucus. The positively charged copolymers showed large size change, and the charge was changed from positive to negative, accompanied by the poor permeability in the mucus. However, the particle size of the hydrophilic copolymers is obviously larger than hydrophilic copolymers, and the permeability of the hydrophilic copolymers is lower in the mucus. We can speculate that this phenomenon may largely facilitate the neutral and hydrophobic copolymers to get access to the mucus layer. Figure 5C shows the permeability of different sizes of copolymers in the mucus layer. Increased particle size correlates with decreased infiltration. The permeability of copolymer A increased rapidly after $2 \mathrm{~h}$. We can speculate that with the increase of particle size, the copolymers are more likely to be trapped by the network structure of mucus. Notably, although the $\zeta$-potential of all the copolymer solutions eventually reached to $-30 \mathrm{mV}$ in the mucus layer, the particle size of electrically neutral and hydrophobic polymers changed much less than that of the others, which may account for the better permeability in the mucus layer. Interestingly, the release rate became slow over time, probably due to the increased particle size caused by the interaction with the mucus.

\section{In vitro cytotoxicity assays}

The cytotoxicity of copolymer solutions against Caco-2 cells was performed by MTT assay, as shown in Figure 6. The cytotoxicity of polymers all increased slightly with the increase in concentration, and the cell viability was $>80 \%$ even at the highest concentration of polymers $(400 \mu \mathrm{g} / \mathrm{mL})$ for $24 \mathrm{~h}$. The results suggested that our materials were essentially non-toxic.

\section{In vitro distribution in the mouse small intestine}

There are many mechanisms of GI mucus, including electrostatic interactions and polymer interpenetration, ${ }^{38-41}$ which are all designed to facilitate adhesion to mucus. In this experiment, by scanning the intestinal from outside

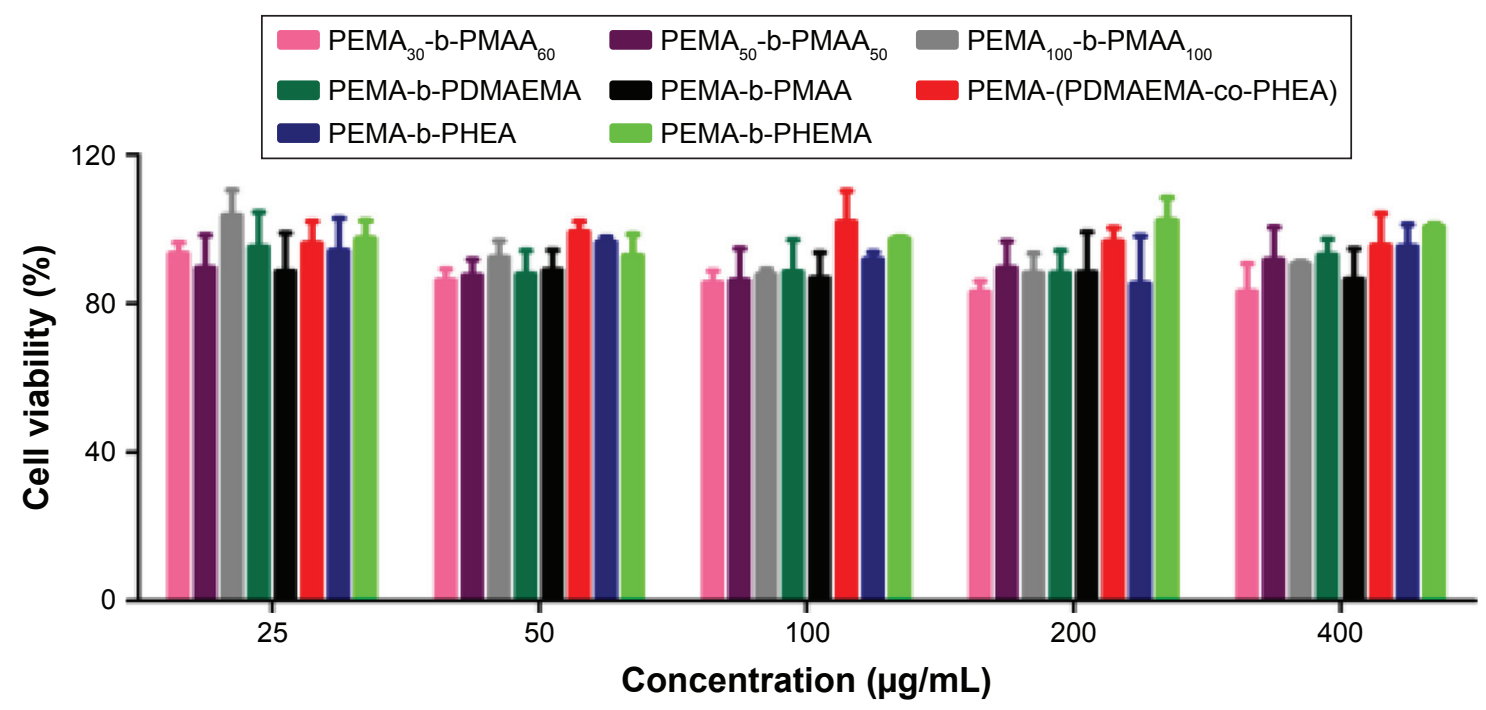

Figure 6 Cytotoxicity test of different polymers in Caco-2 cells after $24 \mathrm{~h}$ of incubation.

Abbreviations: PEMA, poly ethyl methacrylate; PDMAEMA, poly (2-[dimethylamino] ethyl methacrylate); PHEA, poly (2-hydroxyethyl acrylate); PHEMA, poly (2-hydroxyethyl methacrylate); PMAA, poly (methacrylic acid). 
to inside layer by layer with a laser scanning confocal microscope, we could distinguish which copolymers are more likely to pass through the mucous membranes and spread faster in the intestinal. We scanned the intestinal from the outside to the inside every $30 \mu \mathrm{m}$ and observed changes of fluorescence intensity at the same location. In Figure 7, by comparing PEMA-b-PDMAEMA, PEMA-b-PMAA, and PEMA-(PDMAEMA-co-PHEA), it is obvious that PEMA(PDMAEMA-co-PHEA) copolymer showed stronger fluorescence intensity at the same location. It can be inferred that uncharged materials are easier to pass through the mucus layer. Compared with PEMA-b-PHEMA, PEMAb-PHEA can also partly pass through the mucus layer, but obviously, the fluorescence intensity of PEMA-b-PHEMA is higher. And under the same condition, it can be seen that the fluorescence intensity is becoming weaker with the increase of particle size in the same position. We can assume that with increasing size, the particles are less likely to pass through the mucus layer.

Based on these results, the validity of this approach was demonstrated for the permeability of various copolymers. We can directly compare the fluorescence intensity to judge the behavior of polymer in the mucus layer. We think that the mucin fiber interweave to form a dynamic mesh structure with different pore sizes to influence the permeability of materials with different sizes. Therefore, the material with smaller particle size will more easily permeate through the mucus layer, regardless of other factors. ${ }^{23} \mathrm{With}$ regard to the surface charge of the nanoparticles, according to the relevant literature, materials with negative charges are better
(+) PEMA-b-PDMAEMA
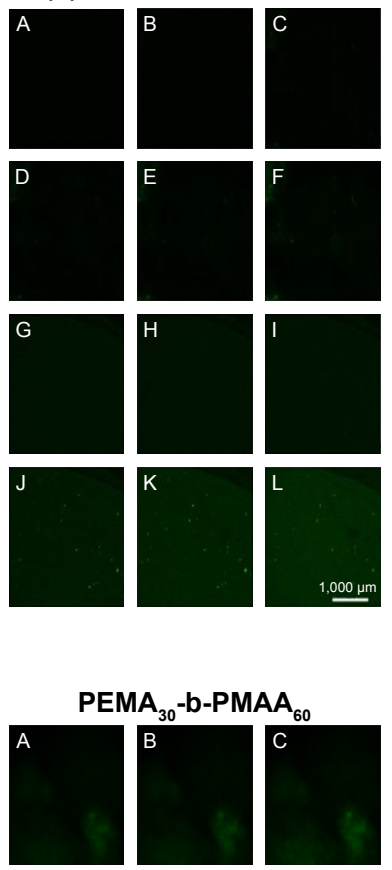

$\mathrm{MA}_{30}-\mathrm{b}-\mathrm{PMAA}_{60}$
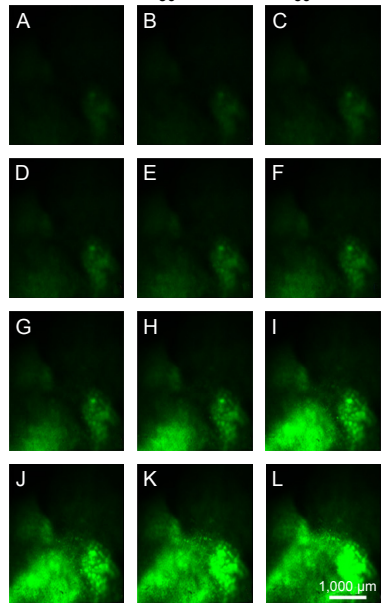

(-) PEMA-b-PMAA
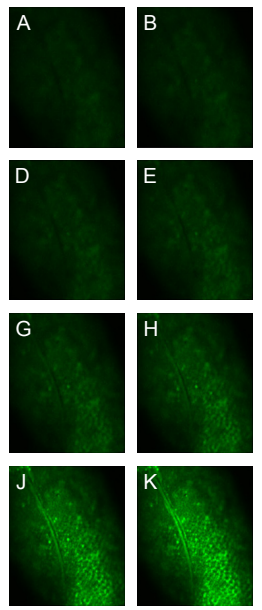

PEMA $_{50}-$ b-PMAA $_{50}$
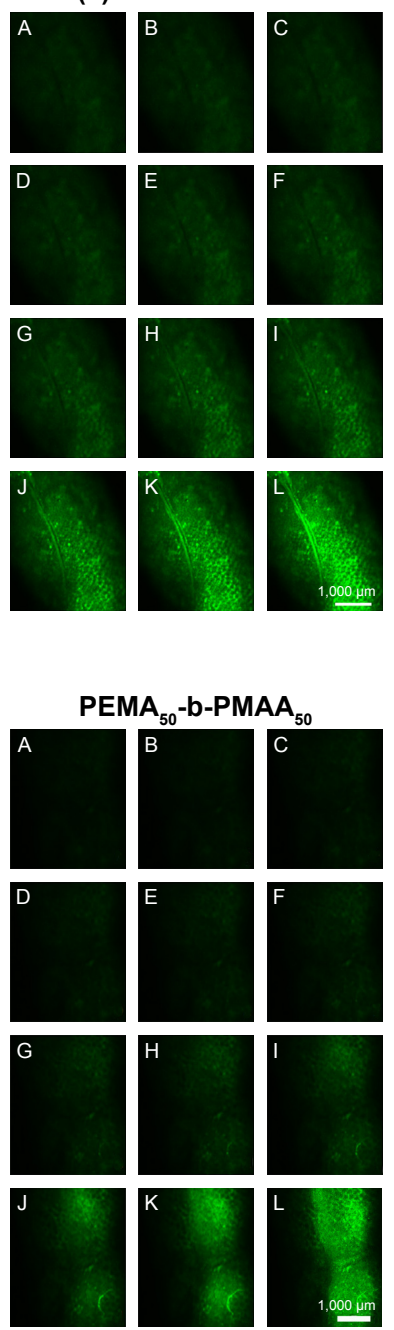

\section{PEMA-(PDMAEMA-CO-PHEA)}
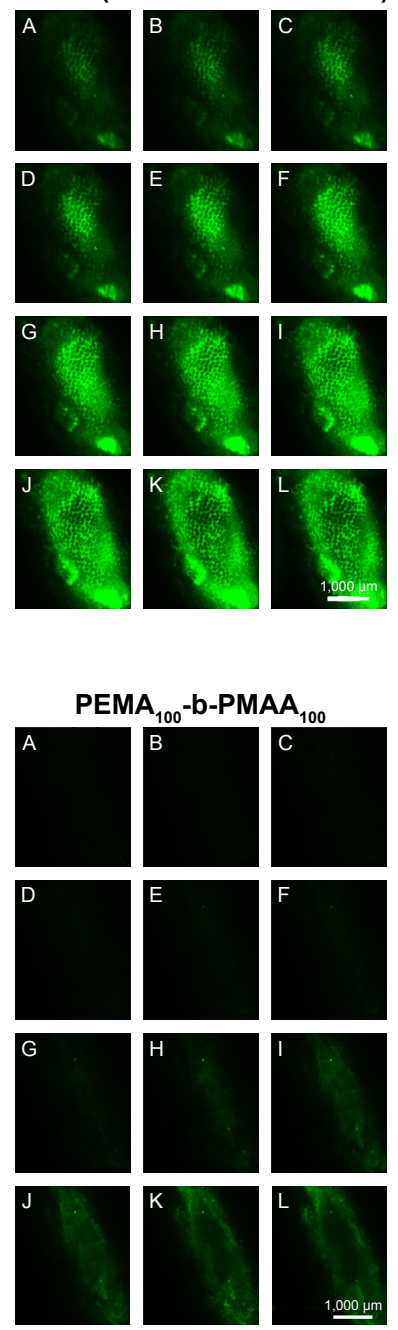

(Hydrophilic) PEMA-b-PHEA
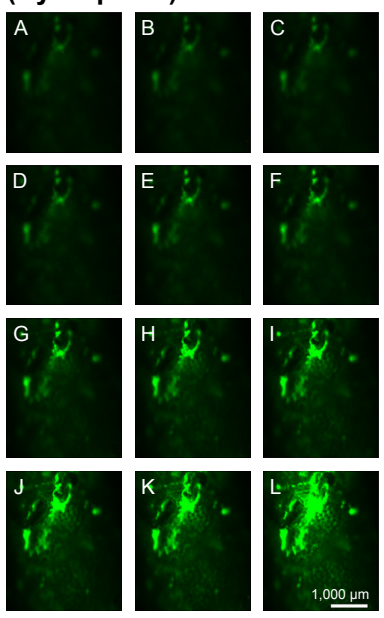

(Hydrophobic)

PEMA-b-PHEMA
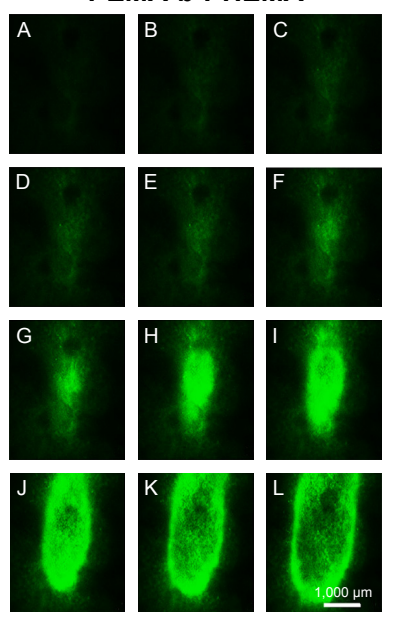

Figure 7 The distribution of different copolymers in intestinal mucus layer.

Notes: The distance interval is $30 \mu \mathrm{m}$ from $A$ to $L$ in each sub-figure. White scale bars indicate $1,000 \mu \mathrm{m}$. Magnification $\times 40$.

Abbreviations: PEMA, poly ethyl methacrylate; PDMAEMA, poly (2-[dimethylamino] ethyl methacrylate); PHEA, poly (2-hydroxyethyl acrylate); PHEMA, poly (2-hydroxyethyl methacrylate); PMAA, poly (methacrylic acid). 
than materials with positive charges in terms of mucus permeability, and the uncharged materials is better than the negatively charged materials. ${ }^{24}$ Regarding the surface hydrophilicity of nanoparticles, according to the discussion above, the hydrophobic domain of mucin fiber may offer stronger interaction with the hydrophobic nanoparticles, prolonging the transport time in the mucus layer. ${ }^{25,42,43}$

\section{In vivo distribution studies}

According to previous reports, ${ }^{33,44}$ nanoparticles can be limited in mucus layer of GI tract from minutes to hours before being cleared by mucus barrier. From the in vitro results of the distribution in the mouse small intestine, we can make a preliminary conclusion that PEMA-(PDMAEMA-coPHEA) and PEMA-b-PHEMA can be easily absorbed and excreted in epithelium. In order to further investigate the effects of different kinds of copolymers in the GI tract, the in vivo distribution studies were carried out. The absorption and excretion of the copolymer solutions in the GI tract indicated the influence of mucus on the copolymers of different properties and sizes. As shown in Figure 8A and C, the absorption and excretion of positively charged sample PEMA-b-PDMAEMA showed no obvious change in the first $8 \mathrm{~h}$, whereas the fluorescence intensity of negatively charged sample PEMA-b-PMAA decreased gradually over time. However, the fluorescence intensity of uncharged copolymers disappeared faster than that of the negatively charged copolymers in Figure 8E. It can be seen from the comparison that the absorption and excretion of uncharged copolymers are superior to negative copolymers over positively charged copolymers in the GI tract. In Figure 8F, the fluorescence intensity of the hydrophilic copolymer PEMA-b-PHEA and hydrophobic copolymer PEMA-b-PHEMA decreased gradually in vivo. The average fluorescence intensity of PEMA-b-PHEMA decreased more rapidly than that of PEMA-b-PHEA from 0.5 to $1 \mathrm{~h}$, but the overall absorption and excretion were not significantly different. These results correlated well with the study of the behavior of the copolymers and indicated which copolymers could be better across to the mucus layer. It should be noted that, when the absorption and excretion on the mucus layer were evaluated, uncharged and hydrophobic copolymers exhibited higher relative bioavailability than other copolymers. This phenomenon might underscore the importance of mucus layer in the in vivo investigation for oral delivery platforms. Moreover, the in vivo pharmacological study showed excellent correlations with the in vitro mucus diffusion. Our results demonstrated that it is a promising strategy to develop nanocarriers that could overcome multiple barriers in oral absorption. Besides, from the images of stomach and intestines, the change of fluorescence intensity of the five copolymers was more obvious. The absorption of the copolymer PEMA-(PDMAEMA-co-PHEA) was significantly higher than that of PEMA-b-PMAA and PEMA-b-PDMAEMA. Compared to PEMA-b-PHEA, the fluorescence intensity of PEMA-b-PHEMA decreased more rapidly. Therefore, we can conclude that the mucus has little effect on PEMA(PDMAEMA-co-PHEA) and PEMA-b-PHEMA. Figure 8B and $\mathrm{D}$ shows the absorption and excretion of copolymers with different sizes in vivo, respectively. In Figure $8 \mathrm{G}$, it can be seen that the average fluorescence intensity of copolymer A disappeared quickly and the signals disappeared at $12 \mathrm{~h}$ postinjection. In contrast, the average fluorescence intensity of copolymers B and C decreased slowly. This is consistent with the changes of the fluorescence intensity in the GI tract. According to the results, the change of fluorescence intensity reflects the absorption and excretion of the copolymers in vivo. It is obvious that the smaller the particle size, the easier it is for the copolymers to pass through the GI mucosa and the faster for the absorption of the copolymers in absorptive epithelium. Moreover, Figure 5D shows that the amount of copolymer-cypate released in vitro was $<6 \%$ in $12 \mathrm{~h}$, indicating that the change of fluorescence intensity in vivo is the cause of copolymer-cypate rather than the free cypate.

\section{Ex vivo distribution in the small intestine}

To further investigate the influence of surface properties and size on the interaction of nanoparticles and mucus layer, ex vivo distribution of different nanoparticles was conducted, as shown in Figure 9. Compared to the negatively charged and uncharged copolymers, positively charged copolymers are trapped in the mucus layer and the average fluorescence intensity outside the mucus layer is lower. In Figure 9A, there is no obvious difference between hydrophilic polymers and hydrophobic polymers. However, the average fluorescence intensity of hydrophobic polymers shown in Figure $9 \mathrm{C}$ is higher than that of hydrophilic polymers, and the average fluorescence intensity of uncharged copolymers is higher than that of negative charged copolymers. In combination with the in vivo results, the ex vivo results indicated that PEMA-(PDMAEMA-co-PHEA) and PEMA-b-PHEMA have stronger ability to pass through the mucus layer. From Figure 9B and D, it is obvious that the smaller the particle size, the easier it is to pass through the mucus layer. These results were consistent with the results of the in vitro studies. 
A

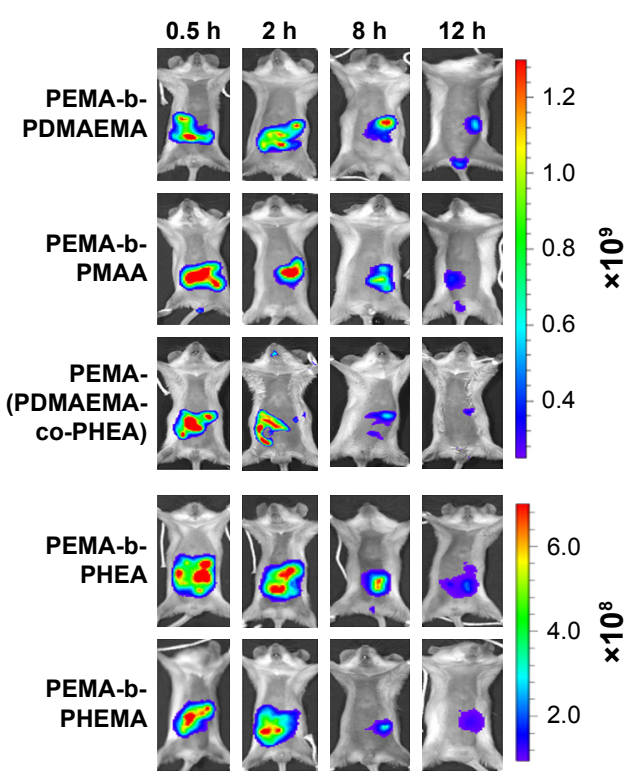

C

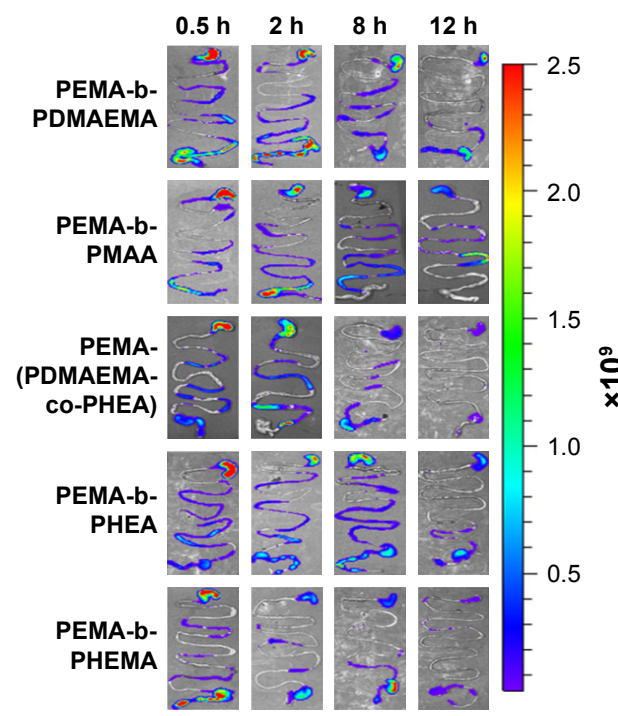

B

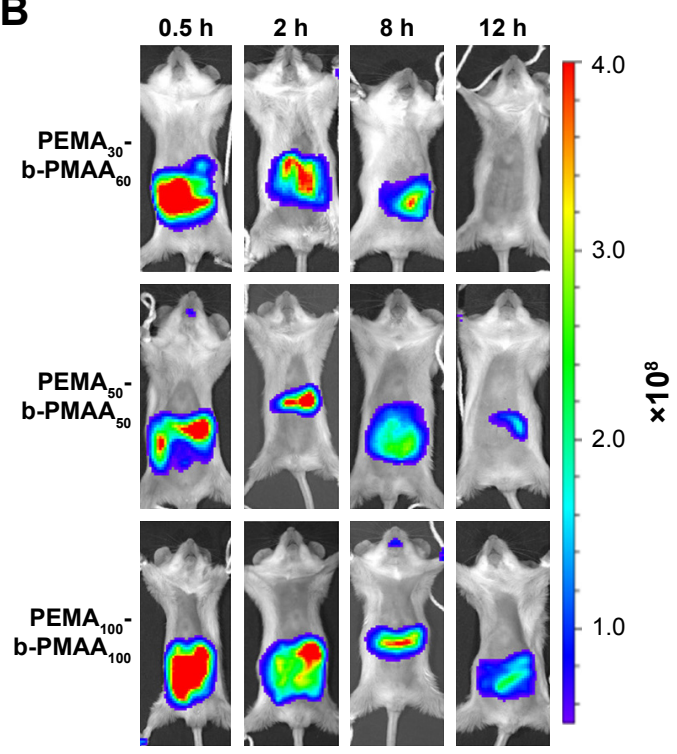

D

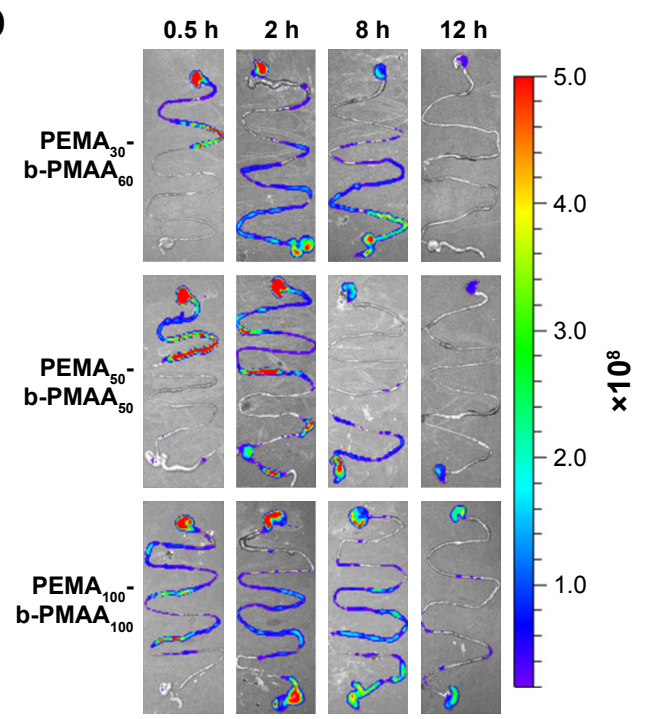

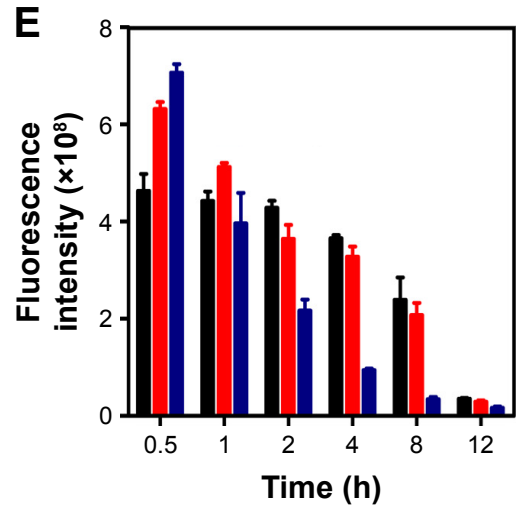

(+) PEMA-b-PDMAEMA

(-) PEMA-b-PMAA

- PEMA-(PDMAEMA-CO-PHEA)

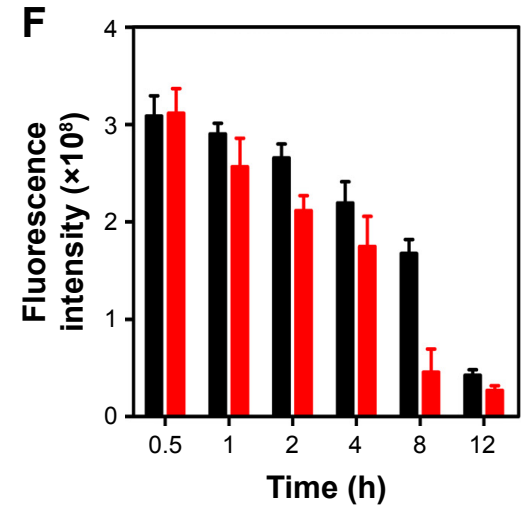

(Hydrophilic) PEMA-b-PHEA (Hydrophobic) PEMA-b-PHEMA

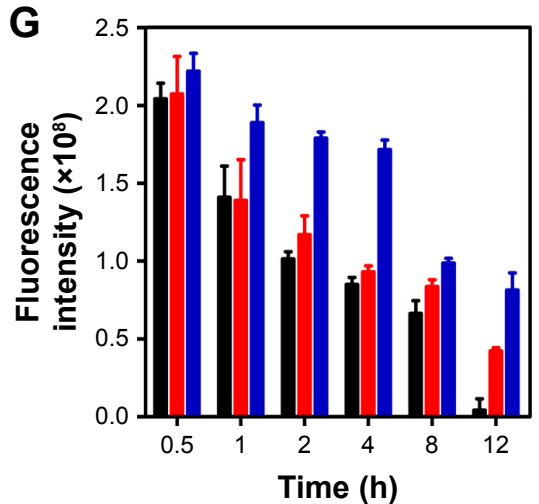

Time (h)

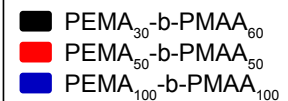

Figure $\mathbf{8}$ (A and $\mathbf{C}$ ) Intragastric administration of five different properties, (B and $\mathbf{D})$ three different size polymer solutions in different time, and variation of average fluorescence intensity of (E) PEMA-b-PDMAEMA/PEMA-b-PMAA/PEMA-(PDMAEMA-co-PHEA), (F) PEMA-b-PHEA/PEMA-b-PHEMA, and (G) three different size polymer solutions.

Abbreviations: PEMA, poly ethyl methacrylate; PDMAEMA, poly (2-[dimethylamino] ethyl methacrylate); PHEA, poly (2-hydroxyethyl acrylate); PHEMA, poly (2-hydroxyethyl methacrylate); PMAA, poly (methacrylic acid). 
A

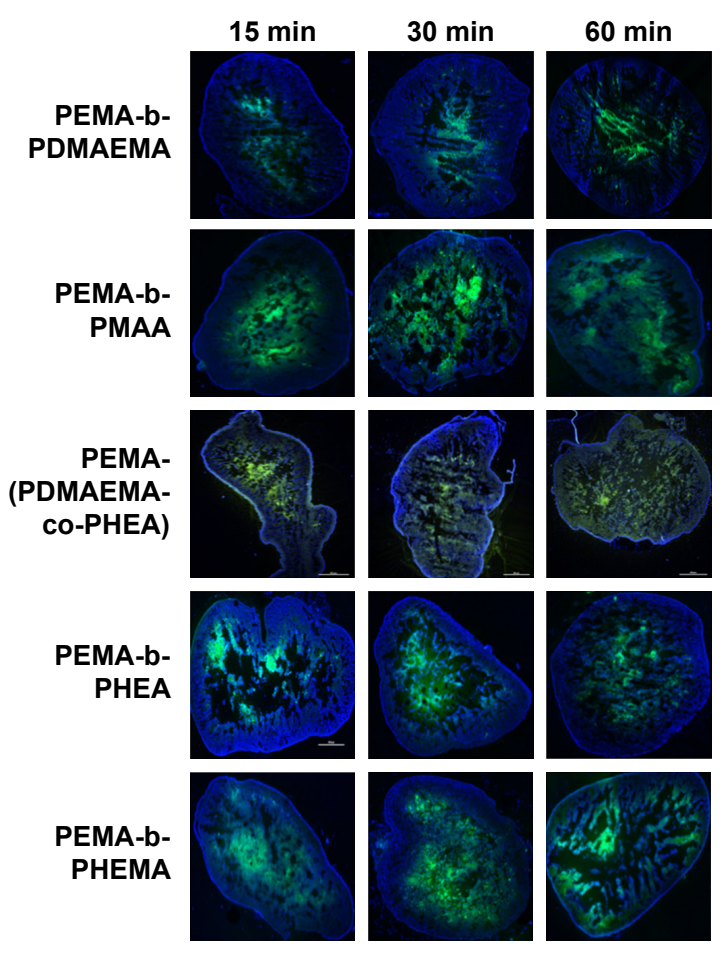

C
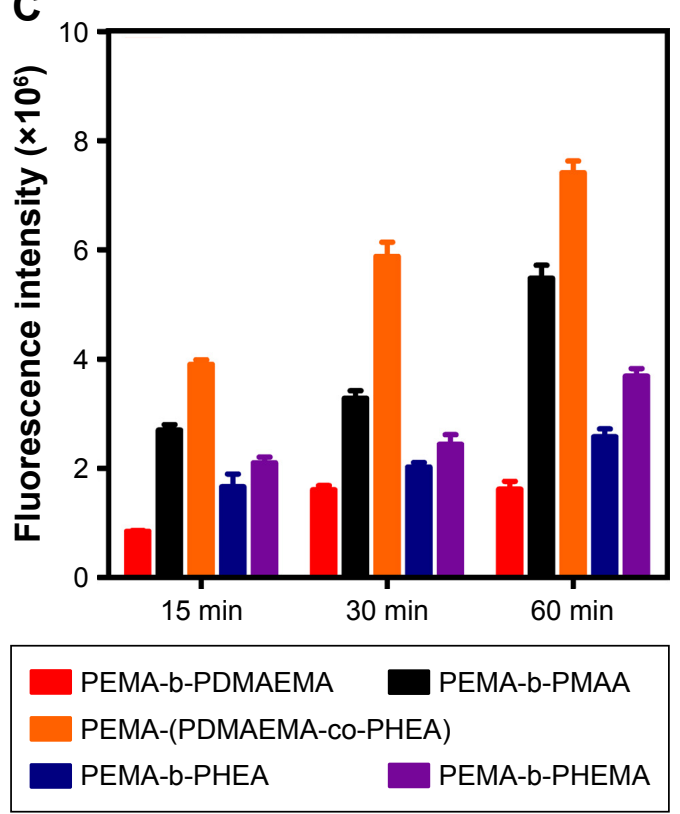

B
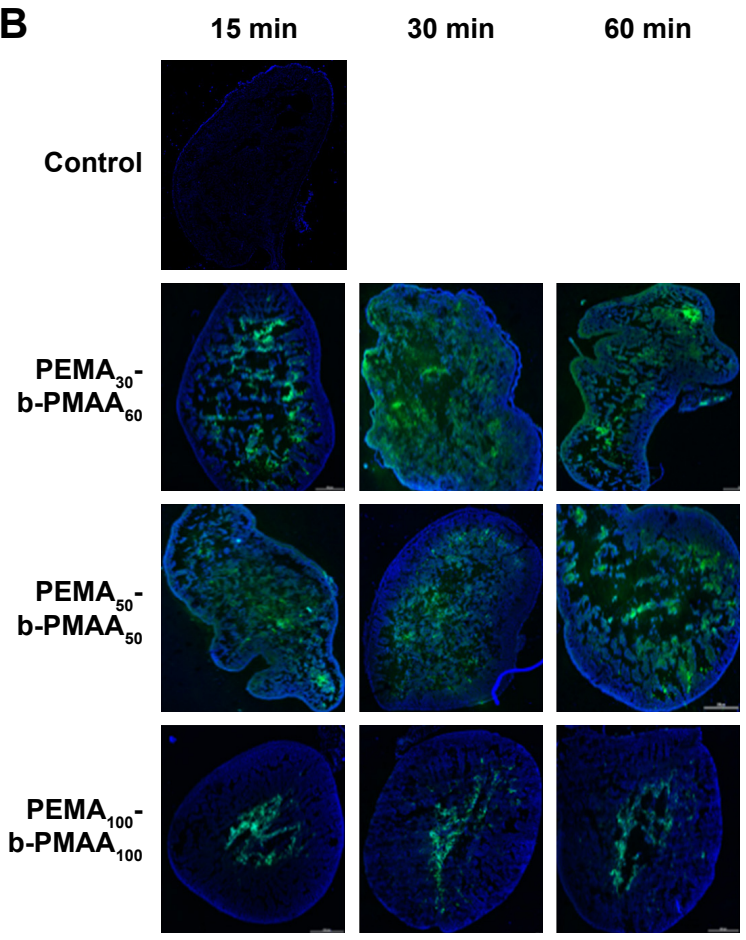

D
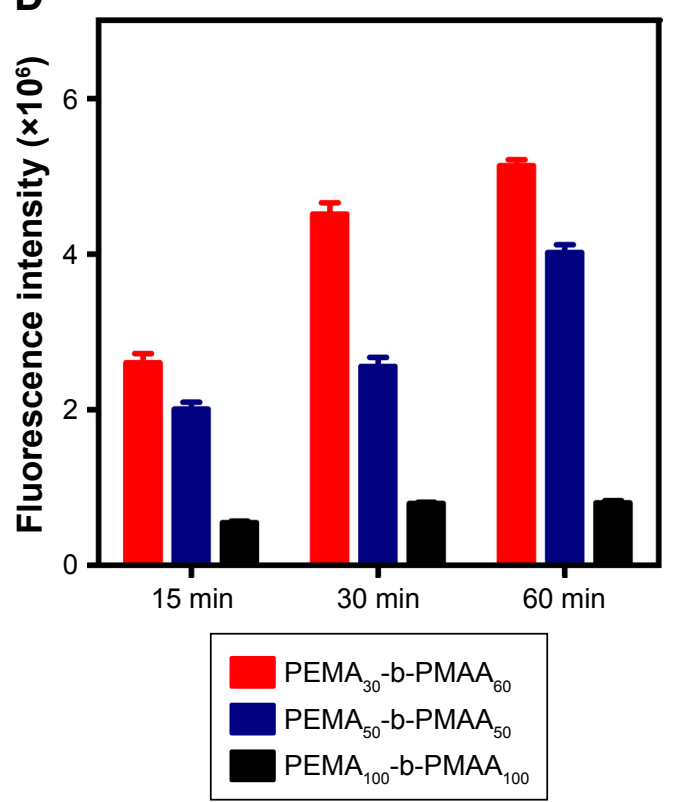

Figure 9 Distribution of $(\mathbf{A})$ different properties copolymers; (B) three different size copolymers in jejunum; average fluorescence intensity of (C) five kinds of copolymers and (D) three different size copolymers in intestinal except mucus layer. Scale bars: $500 \mu \mathrm{m}$. Magnification $\times 40$.

Abbreviations: PEMA, poly ethyl methacrylate; PDMAEMA, poly (2-[dimethylamino] ethyl methacrylate); PHEA, poly (2-hydroxyethyl acrylate); PHEMA, poly (2-hydroxyethyl methacrylate); PMAA, poly (methacrylic acid).

Based on these results, we believe that due to the negatively charged nature of the mucus layer, the negatively charged copolymers are easier to penetrate the mucus layer than positively charged copolymers due to electrostatic interactions. In addition, as compared to the negatively charged copolymers, the uncharged copolymers showed greater permeability in the mucus layer due to the minimal interaction with mucus. ${ }^{42}$ Hydrophilicity is also an important factor. The hydrophobic mucus layer is expected to have stronger interaction with hydrophobic copolymers than hydrophilic ones, and therefore, hydrophobic copolymers are more likely to be absorbed and excreted in the mucus. In our study, we synthesized diblock copolymers by ATRP, which can adjust the particle size easily and obtain different properties 
polymers. We also studied the relationship between the properties of the nanoparticles and permeability in the mucous layer, which provides guidance on the design of nanocarriers for oral administration.

\section{Conclusion}

For oral administration, the mucus layer hinders the effective absorption of drugs. A drug administration will fail if it is unable to reach the epithelial surfaces of the GI tract in a relatively short time. It is therefore important to have a bettering understanding of how physical and chemical properties of drug carriers affect the interaction with mucus layer. In this study, we developed a series of copolymers for in vitro and in vivo experiments and found that hydrophobicity and neutral charge provide copolymers significant advantages in getting across the mucus layer for enhanced drug uptake. These results provide insights into developing optimal nanocarriers for oral administration. In addition, model hydrophobic drug cypate was encapsulated into the copolymers, and the results showed no obvious changes in particle size and $\zeta$-potential, indicating that uploading hydrophobic drugs would not affect the interaction between mucus with polymers. In the future, we will study the transport of drug loaded particles in vivo and in vitro based on the results obtained.

\section{Acknowledgments}

This work was financially supported by the National Natural Science Foundation of China (No 81673360), Shandong Provincial Natural Science Foundation (No ZR2016HM45 and ZR2017BH006), China Postdoctoral Natural Science Foundation (2017M612210), and Scientific Research Foundation for Youth Scholars from Qingdao University (No 41117010026).

\section{Disclosure}

The authors report no conflicts of interest in this work.

\section{References}

1. Roger E, Lagarce F, Garcion E, Benoit JP. Biopharmaceutical parameters to concider in order to alter the fate of nanocarriers after oral delivery. Nanomedicine. 2010;5:287-306.

2. Agrawal AK, Harde H, Thanki K, Jain S. Improved stability and antidiabetic potential of insulin containing folic acid functionalized polymer stabilized multilayered liposomes following oral administration. Biomacromolecules. 2014;15:350-360.

3. Joshi N, Saha R, Shanmugam T, Balakrishnan B, More P, Banerjee R. Carboxymethyl-chitosan-tethered lipid vesicles: hybrid nanoblanket for oral delivery of paclitaxel. Biomacromolecules. 2013;14:2272-2282.

4. Hunter AC, Elsom J, Wibroe PP, Moghimi SM. Polymeric particulate technologies for oral drug delivery and targeting: a pathophysiological perspective. Nanomedicine. 2012;8:S5-S20.
5. Ensign LM, Cone R, Hanes J. Oral drug delivery with polymeric nanoparticles: the gastrointestinal mucus barriers. Adv Drug Deliv Rev. 2012;64:557-570.

6. Bernkop-Schnürch A. Nanocarrier systems for oral drug delivery: do we really need them? Eur J Pharm Sci. 2013;49:272-277.

7. Authimoolam SP, Vasilakes AL, Shah NM, et al. Synthetic oral mucin mimic from polymer micelle networks. Biomacromolecules. 2014;15: 3099-3111.

8. Ensign LM, Henning A, Schneider CS, et al. Ex vivo characterization of particle transport in mucus secretions coating freshly excised mucosal tissues. Mol Pharm. 2013;10:2176-2182.

9. Plapied L, Duhem N, des Rieux A, Préat V. Fate of polymeric nanocarriers for oral drug delivery. Curr Opin Colloid Interf Sci. 2011;16: $228-237$.

10. Goycoolea FM, Menchicchi B, Caramella CM, et al. Innovative methods and applications in mucoadhesion research. Macromol Biosci. 2017; 17(8).1600534.

11. Boegh M, Nielsen HM. Mucus as a barrier to drug delivery - understanding and mimicking the barrier properties. Basic Clin Pharmacol. 2015;116:179-186.

12. Chater PI, Wilcox MD, Pearson JP. Efficacy and safety concerns over the use of mucus modulating agents for drug delivery using nanoscale systems. Adv Drug Deliv Rev. 2018;124:184-192.

13. Yang T, Wei SJ, Zhou CM, et al. Mucus adhesion- and penetrationenhanced liposomes for paclitaxel oral delivery. Int J Pharm. 2018;537: 245-256.

14. Grießinger J, Dünnhaupt S, Cattoz B, et al. Methods to determine the interactions of micro- and nanoparticles with mucus. Eur $J$ Pharm Biopharm. 2015;96:464-476.

15. Lai SK, Wang Y, Hanes J. Mucus-penetrating nanoparticles for drug and gene delivery to mucosal tissues. Adv Drug Deliv Rev. 2009;61: $158-171$.

16. Crater JS, Carrier RL. Barrier properties of gastrointestinal mucus to nanoparticle transport. Macromol Biosci. 2010;10:1473-1483.

17. Khutoryanskiy VV. Beyond PEGylation: alternative surface-modification of nanoparticles with mucus-inert biomaterials. Adv Drug Deliv Rev. 2018;124:140-149.

18. Yun Y, Cho YW, Park K. Nanoparticles for oral delivery: targeted nanoparticles with peptidic ligands for oral protein delivery. Adv Drug Deliv Rev. 2013;65:822-832.

19. des Rieux A, Fievez V, Garinot M, Schneider YJ, Préat V. Nanoparticles as potential oral delivery systems of proteins and vaccines: a mechanistic approach. J Control Release. 2006;116:1-27.

20. Strugala V, Allen A, Dettmar PW, Pearson JP. Colonic mucin: methods of measuring mucus thickness. Proc Nutr Soc. 2003;62:237-243.

21. Tahara K, Nishio M, Takeuchi H. Evaluation of liposomal behavior in the gastrointestinal tract after oral administration using real-time in vivo imaging. Drug Dev Ind Pharm. 2018;44(4):608-614.

22. Desai D, Prabhakar N, Mamaeva V, et al. Targeted modulation of cell differentiation in distinct regions of the gastrointestinal tract via oral administration of differently PEG-PEI functionalized mesoporous silica nanoparticles. Int J Nanomedicine. 2016;11:299-313.

23. He C, Hu Y, Yin L, Tang C, Yin C. Effects of particle size and surface charge on cellular uptake and biodistribution of polymeric nanoparticles. Biomaterials. 2010;31:3657-3666

24. Murgia X, Pawelzyk P, Schaefer UF, Wagner C, Willenbacher N, Lehr CM. Size-limited penetration of nanoparticles into porcine respiratory mucus after aerosol deposition. Biomacromolecules. 2016;17: 1536-1542.

25. Maisel K, Ensign L, Reddy M, Cone R, Hanes J. Effect of surface chemistry on nanoparticle interaction with gastrointestinal mucus and distribution in the gastrointestinal tract following oral and rectal administration in the mouse. J Control Release. 2015;197:48-57.

26. Sheng J, Han L, Qin J, et al. N-Trimethyl chitosan chloride-coated PLGA nanoparticles overcoming multiple barriers to oral insulin absorption. ACS Appl Mater Interfaces. 2015;7:15430-15441. 
27. Liu M, Zhang J, Zhu X, et al. Efficient mucus permeation and tight junction opening by dissociable "mucus-inert" agent coated trimethyl chitosan nanoparticles for oral insulin delivery. J Control Release. 2016; 222:67-77.

28. Bourganis V, Karamanidou T, Samaridou E, Karidi K, Kammona O, Kiparissides C. On the synthesis of mucus permeating nanocarriers. Eur J Pharm Biopharm. 2015;97:239-249.

29. Pereira De Sousa I, Steiner C, Schmutzler M, et al. Mucus permeating carriers: formulation and characterization of highly densely charged nanoparticles. Eur J Pharm Biopharm. 2015;97:273-279.

30. Sheng J, He H, Han L, et al. Enhancing insulin oral absorption by using mucoadhesive nanoparticles loaded with LMWP-linked insulin conjugates. J Control Release. 2016;233:181-190.

31. Zhang Y, Du X, Zhang Y, et al. Thiolated eudragit-based nanoparticles for oral insulin delivery: preparation, characterization, and evaluation using intestinal epithelial cells in vitro. Macromol Biosci. 2014;14: 842-852.

32. Robinson KL, Khan MA, de Paz Bez MV, Wang S, Armes SP. Controlled polymerization of 2-hydroxyethyl methacrylate by ATRP at ambient temperature. Macromolecules. 2001;34:3155-3158.

33. Weaver JVM, Bannister I, Robinson KL, Bories-Azeau X, Armes SP, Smallridge M. Stimulus-responsive water-soluble polymers based on 2-hydroxyethyl methacrylate. Macromolecules. 2004;37:2395-2403.

34. Zhang W, Li Y, Liu L, et al. Amphiphilic toothbrushlike copolymers based on poly(ethylene glycol) and poly( $\varepsilon$-caprolactone) as drug carriers with enhanced properties. Biomacromolecules. 2010;11: 1331-1338.
35. Ibrahim BM, Park S, Han B, Yeo Y. A strategy to deliver genes to cystic fibrosis lungs: a battle with environment. J Control Release. 2011;155: 289-295.

36. D Angelo I, Casciaro B, Miro A, Quaglia F, Mangoni ML, Ungaro F. Overcoming barriers in Pseudomonas aeruginosa lung infections: engineered nanoparticles for local delivery of a cationic antimicrobial peptide. Colloids Surf B Biointerfaces. 2015;135:717-725.

37. Primard C, Rochereau N, Luciani E, et al. Traffic of poly(lactic acid) nanoparticulate vaccine vehicle from intestinal mucus to sub-epithelial immune competent cells. Biomaterials. 2010;31:6060-6068.

38. Cu Y, Saltzman WM. Mathematical modeling of molecular diffusion through mucus. Adv Drug Deliv Rev. 2009;61:101-114.

39. Cone RA. Barrier properties of mucus. Adv Drug Deliv Rev. 2009;61: 75-85.

40. Peppas NA, Huang Y. Nanoscale technology of mucoadhesive interactions. Adv Drug Deliv Rev. 2004;56:1675-1687.

41. Peppas NA. Molecular calculations of poly(ethylene glycol) transport across a swollen poly(acrylic acid)/mucin interface. J Biomater $\mathrm{Sci}$ Polym Ed. 1998;9:535-542.

42. Shan W, Zhu X, Liu M, et al. Overcoming the diffusion barrier of mucus and absorption barrier of epithelium by self-assembled nanoparticles for oral delivery of insulin. ACS Nano. 2015;9:2345-2356.

43. Lai SK, Wang Y, Wirtz D, Hanes J. Micro- and macrorheology of mucus. Adv Drug Deliv Rev. 2009;61:86-100.

44. Gaucher G, Satturwar P, Jones M, Furtos A, Leroux JC. Polymeric micelles for oral drug delivery. Eur J Pharm Biopharm. 2010;76: $147-158$. 


\section{Supplementary materials}
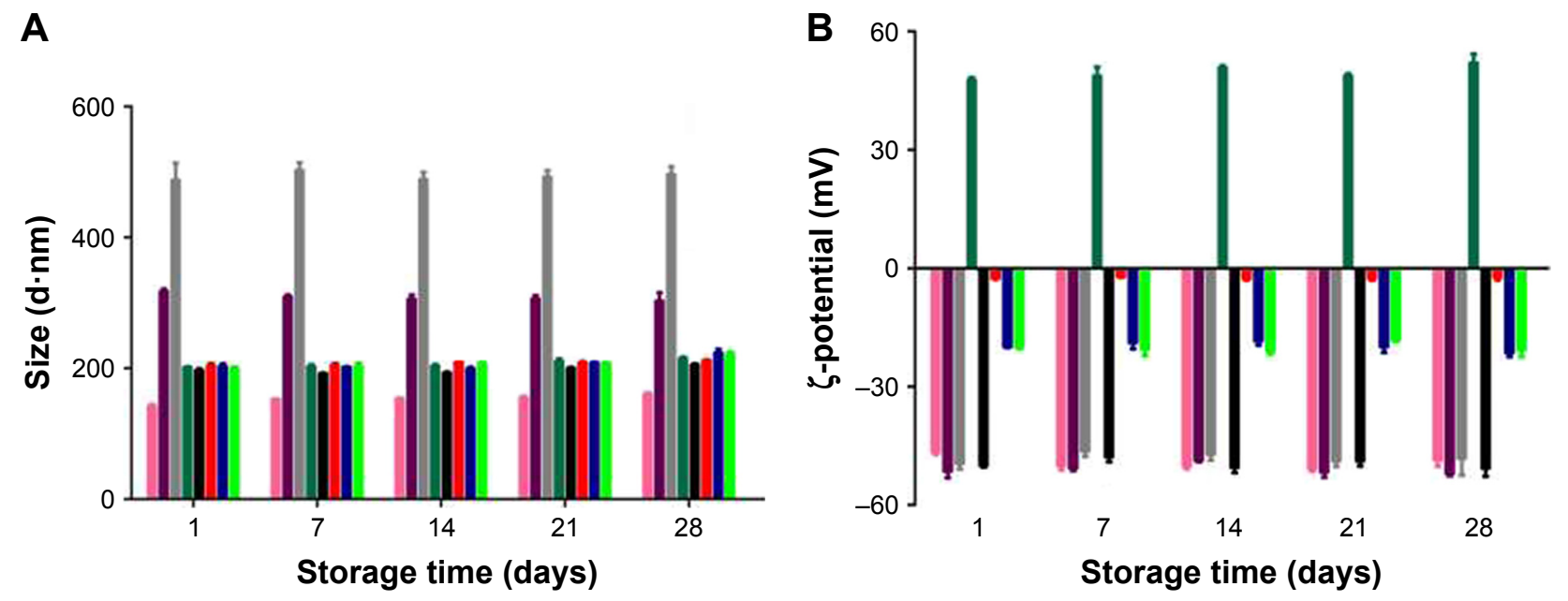

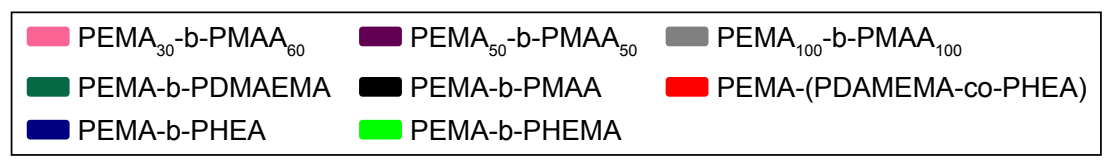

Figure SI (A) Size and (B) $\zeta$-potential of the different polymer solutions after keeping in storage for different time $(n=3)$.

Note: The size and $\zeta$-potential before storage served as the control.

Abbreviations: PEMA, poly ethyl methacrylate; PDMAEMA, poly (2-[dimethylamino] ethyl methacrylate); PHEA, poly (2-hydroxyethyl acrylate); PHEMA, poly (2-hydroxyethyl methacrylate); PMAA, poly (methacrylic acid).
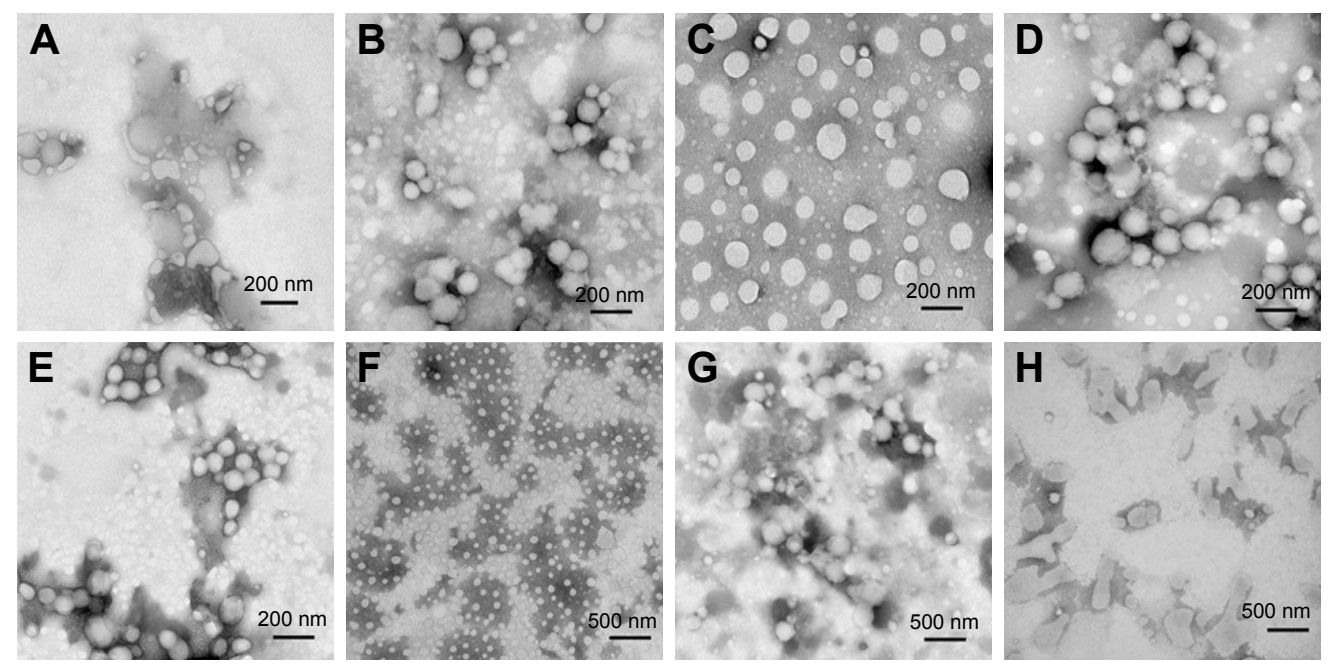

Figure S2 Transmission electron microscope pictures of (A) PEMA-b-PDMAEMA, (B) PEMA-b-PMAA, (C) PEMA-(PDAMEMA-co-PHEA), (D) PEMA-b-PHEA, (E) PEMAb-PHEMA, (F) PEMA 30 -b-PMAA 6 , (G) PEMA ${ }_{50}$-b-PMAA ${ }_{50}$, and (H) PEMA 100 -b-PMAA 100 polymer solutions in mucus.

Abbreviations: PEMA, poly ethyl methacrylate; PDMAEMA, poly (2-[dimethylamino] ethyl methacrylate); PHEA, poly (2-hydroxyethyl acrylate); PHEMA, poly (2-hydroxyethyl methacrylate); PMAA, poly (methacrylic acid).

\section{Publish your work in this journal}

The International Journal of Nanomedicine is an international, peerreviewed journal focusing on the application of nanotechnology in diagnostics, therapeutics, and drug delivery systems throughout the biomedical field. This journal is indexed on PubMed Central, MedLine, CAS, SciSearch ${ }^{\circledR}$, Current Contents $® /$ Clinical Medicine,
Journal Citation Reports/Science Edition, EMBase, Scopus and the Elsevier Bibliographic databases. The manuscript management system is completely online and includes a very quick and fair peer-review system, which is all easy to use. Visit http://www.dovepress.com/ testimonials.php to read real quotes from published authors. 